\title{
27. PETROCHEMISTRY OF BASALTS AND DISTRIBUTION OF ORGANIC GASES: HOLES 407, 408, 409, 410A, 411, 412, and 413, DSDP LEG 49
}

\author{
B. P. Zolotarey, D. Ya. Choporov, and G. I. Voitov, Geological Institute of the USSR Academy of Sciences, \\ Moscow, USSR
}

\section{INTRODUCTION}

This paper considers the petrochemistry of basalts and distribution of organic gases in basalts recovered from Holes $407,408,409,410,410 \mathrm{~A}, 411,412 \mathrm{~A}$, and 413. For this purpose, we compiled variation diagrams for the major rock-forming elements and some petrochemical coefficients, then grouped the plotted values in all diagrams into three mutually overlapping fields corresponding to the three regions of the Mid-Atlantic Ridge in which the DSDP drilling was conducted.

Petrochemical differences of basalts developed in various regions of the Mid-Atlantic Ridge can be explained either by lateral heterogeneity of the composition of the mantle substratum, or by the various depths of magma generation. The absence of a discrete distribution of the major rock-forming elements (especially aluminum, magnesium, and titanium) in the basalts of each of the three identified groups testifies to a negligible role of crystallization differentiation. This does not contradict the petrographic characteristics of the basalts.

The distribution of organic gases in basalts is extremely diverse. We did not establish a distinct correlative dependence between the various gases and the chemistry of basalts in this study.

\section{ANALYTICAL PROCEDURE}

Chemical analyses of the samples were performed in the Chemical-Analytical Laboratory of the Geological Institute of the USSR Academy of Sciences, Moscow. In accordance with the previously described methods (Zolotarev et al., in press), the summary weight of the material taken for analysis was, as a rule, 3.5 grams. The results obtained were averaged from two parallel determinations in which the oxide sum composing the materials under study was 99.5 to 100.5 per cent.

To evaluate the reproducibility and accuracy of the results, the samples were analyzed simultaneously with samples recommended as interlaboratory standards by the French Scientific Center on Petrographic and Geochemical Studies (Roubault et al., 1970) and the National Association of Technical Studies of France (de La Roche and Govindaraju, 1973).

The results of analysis of the standard basalt samples BR and diorite DR-N, noted above, and the data obtained from our samples are presented in Table 1 .

A comparison of the values obtained shows that the most significant disagreements in the results of the chemical analyses were $\mathrm{Fe}_{2} \mathrm{O}_{3}$ and the alkaline elements in basalt BR, and for the alkaline elements in diorite DR-N. The analytical results of the standard samples are in good agreement with the recommended values, and within the limits of relative deviations from the control analyses and from the average values of the element contents in the standard samples of basalt BR (Figure 1) and diorite DR-N (Figure 2).

The study of the composition of organic gases necessitates obtaining extracts from basalts. Gas extracts were obtained from small weights ( 1.25 to $3.5 \mathrm{~g}$ ) that had been mechanically powdered in steel chambers filled with argon. This method of gas extraction proved efficient in previous studies (Zolotarev et al., in press). Nevertheless, there are limitations to this extraction method. Small volumes of gas extracted through powdering small rock weights repeatedly become diluted in an argon-filled chamber $\left(25 \mathrm{~cm}^{3}\right)$, so one cannot always accurately determine the content of a particular component of low-boiling gases $\left(\mathrm{CO}_{2}\right.$, first of all $)$ by means of chromatographic analysis, owing to a limited sensitivity of the catarometer. This results from the amount of the component in the mixture studied being below the threshold response of the detector. The use of highly sensitive flame-ionization detectors for analysis of hydrocarbons eliminates these limitations. A second limitation of the gas extraction method is a lower gas output from powdered rocks

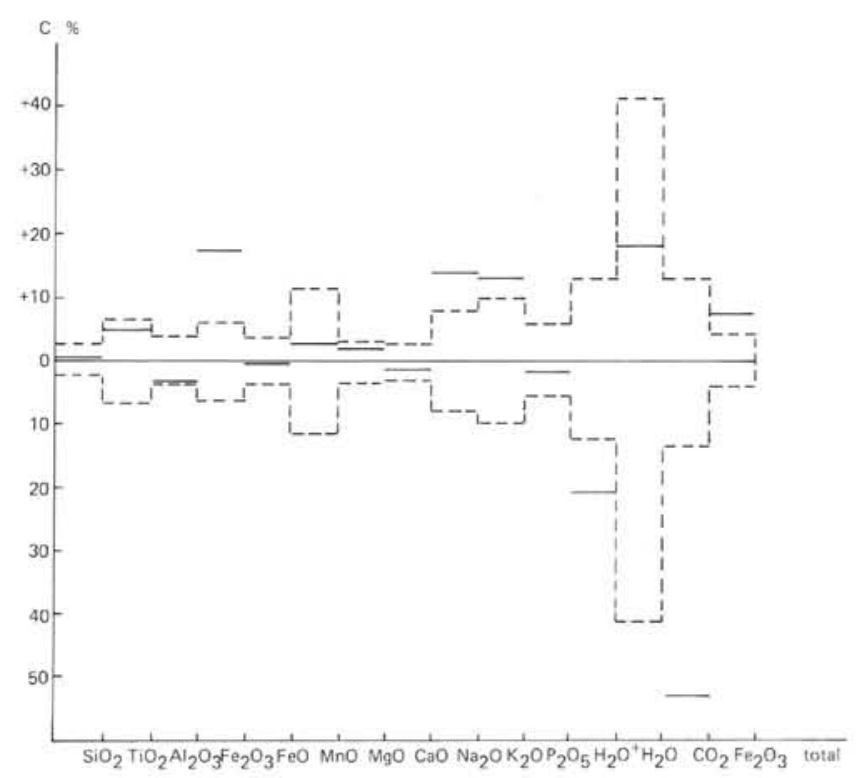

Figure 1. Intervals of relative deviations of carbon percentage for basalt BR. Dotted line indicates relative deviations of interlaboratory results; continuous line shows relative deviations of control analyses performed in the chemical-analytical laboratory of the Geological Institute of the USSR Academy of Sciences. 
TABLE 1

Results of the Analyses of Standard Samples of Basalt BR and Diorite DR-N

\begin{tabular}{|c|c|c|c|c|c|c|c|c|c|c|c|c|c|c|c|c|c|c|c|c|c|c|c|c|c|c|}
\hline \multicolumn{14}{|c|}{ Basalt BR } & \multicolumn{13}{|c|}{ Diorite DR-N } \\
\hline \multirow[t]{2}{*}{$\begin{array}{r}\text { Compo- } \\
\text { nents }\end{array}$} & \multicolumn{6}{|c|}{$\begin{array}{l}\text { Data of the "Centre de Recherches Petrographiques } \\
\text { et Geochimiques" (C.R.P.G.). } \\
\text { M. Roubault, H. de la Roche, and K. Govindaraju } \\
\text { (1970). }\end{array}$} & \multicolumn{5}{|c|}{$\begin{array}{l}\text { Results of the chemical analyses of the stan- } \\
\text { dard sample performed by chemical- } \\
\text { laboratory of the Geological Institute } \\
\text { of the USSR Academy of Sciences }\end{array}$} & \multicolumn{2}{|c|}{$\begin{array}{l}\text { Deviation of } \\
\text { the results of } \\
\text { the analyses }\end{array}$} & \multicolumn{6}{|c|}{$\begin{array}{l}\text { Data of "De L'Association Nationale de la } \\
\text { Recherche Technique" H. de la Roche and K. } \\
\text { Govindaraju (1973) }\end{array}$} & \multicolumn{5}{|c|}{$\begin{array}{l}\text { Results of the chemical analyses of the } \\
\text { standard sample performed by chemical ana- } \\
\text { ly tical laboratory of the Geological Institute } \\
\text { USSR Academy of Sciences }\end{array}$} & \multicolumn{2}{|c|}{$\begin{array}{l}\text { Deviation of } \\
\text { the results of } \\
\text { the analyses }\end{array}$} \\
\hline & $\begin{array}{l}\text { Recom- } \\
\text { mended } \\
\text { Values }\end{array}$ & $\mathrm{d} x$ & $\mathrm{~s}$ & $\mathrm{C} \%$ & $\begin{array}{l}X- \\
S\end{array}$ & $\begin{array}{l}\mathrm{X}+ \\
\mathrm{s}\end{array}$ & $x$ & $\mathrm{~s}$ & c & $\underset{\text { min }}{\mathrm{X}}$ & $\underset{\max }{\mathrm{X}}$ & X-R.V & $\mathrm{C} \%$ & $\begin{array}{l}\text { Recom- } \\
\text { mended } \\
\text { Values }\end{array}$ & $x$ & $s$ & $\mathrm{C} \%$ & $\begin{array}{c}x- \\
S\end{array}$ & $\begin{array}{c}X+ \\
S\end{array}$ & s & $\mathrm{s}$ & c & $\underset{\min }{\mathrm{X}}$ & $\underset{\max }{\mathrm{X}}$ & X-R.V & $\mathrm{C} \%$ \\
\hline $\mathrm{SiO}_{2}$ & 38.20 & 38.75 & 0.98 & 2.5 & 37.77 & 39.73 & 38.33 & 0.09 & 0.02 & 38.24 & 38.42 & +0.13 & 0.3 & 52.75 & 52.91 & 0.86 & 1.6 & 52.05 & 53.77 & 52.13 & 0.20 & 0.38 & 51.93 & 52.33 & -0.42 & \\
\hline $\mathrm{TiO}_{2}$ & 2.60 & 2.61 & 0.17 & 6.5 & 2.44 & 2.78 & 2.72 & 0.0 & 0.0 & 2.72 & 2.72 & +0.12 & 4.6 & 1.10 & 1.10 & 0.12 & 11.0 & 0.96 & 1.20 & 1.12 & 0.02 & 1.8 & 1.10 & 1.14 & +0.02 & 1.8 \\
\hline $\mathrm{Al}_{2} \mathrm{O}_{3}$ & 10.20 & 10.28 & 0.10 & 3.9 & 9.88 & 10.68 & 9.88 & 0.5 & 5.0 & 9.50 & 10.61 & -0.32 & 3.1 & 17.52 & 17.41 & 0.64 & 3.7 & 16.77 & 18.05 & 17.39 & 0.45 & 2.6 & 16.94 & 17.84 & -0.13 & 0.7 \\
\hline $\mathrm{Fe}_{2}^{2} \mathrm{O}_{3}$ & 5.58 & 5.69 & 0.36 & 6.3 & 5.33 & 6.05 & 6.57 & 0.55 & 8.3 & 5.84 & 7.08 & +0.99 & 17.7 & 3.77 & 3.91 & 0.48 & 12.3 & 3.43 & 4.39 & 3.55 & 0.52 & 14.6 & 3.03 & 4.07 & -0.22 & \\
\hline $\mathrm{FeO}$ & 6.57 & 6.32 & 0.26 & 4.0 & 6.26 & 6.78 & 6.56 & 0.12 & 1.8 & 6.44 & 6.68 & -0.01 & 0.15 & 5.31 & 5.34 & 0.29 & 5.4 & 5.05 & 5.63 & 5.21 & 0.02 & 0.4 & 5.19 & 5.23 & -0.10 & 1.9 \\
\hline $\mathrm{MnO}$ & 0.20 & 0.20 & 0.023 & 11.5 & 0.177 & 0.223 & 0.205 & 0.005 & 2.4 & 0.20 & 0.21 & +0.005 & 2.5 & 0.21 & 0.21 & 0.026 & 12.6 & 0.18 & 0.24 & 0.215 & 0.005 & 2.3 & 0.21 & 0.22 & +0.005 & 52.4 \\
\hline $\mathrm{MgO}$ & 13.28 & 13.20 & 0.42 & 3.2 & 12.78 & 13.62 & 13.53 & 0.155 & 1.1 & 13.38 & 13.69 & +0.25 & 1.9 & 4.46 & 4.45 & 0.30 & 6.7 & 4.15 & 4.75 & 4.34 & 0.13 & 3.0 & 4.21 & 4.47 & -0.12 & 2.7 \\
\hline $\mathrm{CaO}$ & 13.80 & 13.78 & 0.35 & 3.1 & 13.43 & 14.13 & 13.69 & 0.01 & 0.07 & 13.68 & 13.70 & -0.11 & 0.8 & 7.07 & 7.04 & 0.24 & 3.4 & 6.80 & 7.28 & 7.17 & 0.03 & 0.4 & 7.14 & 7.20 & +0.10 & 1.4 \\
\hline $\mathrm{Na}_{2} \mathrm{O}$ & 3.05 & 3.12 & 0.25 & 8.0 & 2.87 & 3.37 & 3.49 & 0.14 & 4.0 & 3.35 & 3.63 & +0.44 & 14.4 & 2.99 & 2.98 & 0.21 & 7.0 & 2.77 & 3.19 & 3.30 & 0.12 & 4.0 & 3.18 & 3.42 & +0.311 & 10.4 \\
\hline $\mathrm{K}_{2} \mathrm{O}$ & 1.40 & 1.42 & 0.14 & 9.9 & 1.28 & 1.56 & 1.59 & 0.08 & 5.0 & 1.51 & 1.67 & +0.19 & 13.6 & 1.73 & 1.72 & 0.12 & 6.9 & 1.60 & 1.84 & 1.92 & 0.10 & 5.0 & 1.82 & 2.02 & +0.191 & 11.0 \\
\hline $\mathrm{P}_{2}^{2} \mathrm{O}_{5}$ & 1.04 & 1.05 & 0.06 & 5.7 & 0.99 & 1.11 & 1.025 & 0.005 & 0.5 & 1.02 & 1.03 & -0.015 & 1.4 & 0.25 & 0.26 & 0.05 & 18.0 & 0.21 & 0.31 & 0.27 & 0.00 & 0.00 & 0.27 & 0.27 & +0.02 & 8.0 \\
\hline $\mathrm{H}_{2} \mathrm{O}^{+}$ & 2.30 & 2.31 & 0.29 & 12.6 & 2.02 & 2.60 & 1.81 & 0.04 & 2.2 & 1.77 & 1.85 & -0.49 & 21.3 & 2.19 & 2.24 & 0.23 & 10.2 & 2.01 & 2.47 & 1.96 & 0.023 & 1.3 & 1.94 & 1.99 & -0.23 । & 10.5 \\
\hline $\mathrm{H}_{2} \mathrm{O}^{-}$ & 0.50 & 0.46 & 0.19 & 41.3 & 0.27 & 0.65 & 0.59 & 0.03 & 5.0 & 0.56 & 0.62 & +0.09 & 18 & 0.24 & 0.25 & 0.08 & 33.2 & 0.17 & 0.33 & 0.36 & 0.00 & 0.00 & 0.36 & 0.36 & +0.125 & 50 \\
\hline \multirow{2}{*}{$\mathrm{CO}_{2}$} & 0.86 & 0.84 & 0.11 & 13.1 & .073 & 0.95 & 0.40 & 0.25 & 62.5 & 0.15 & 0.65 & -0.46 & 53.5 & 0.15 & 0.15 & 0.06 & 40 & 0.09 & 0.21 & 0.22 & 0.07 & 34 & 0.15 & 0.30 & +0.074 & 47 \\
\hline & 99.58 & 100.23 & 4.00 & 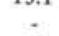 & 96.23 & 104.23 & 100.4 & 1.98 & . & 98.36 & 100.56 & . & . & 99.74 & 99.95 & 3.786 & . & 96.24 & 103.66 & 99.16 & 1.69 & & 97.47 & 100.86 & . & . \\
\hline $\begin{array}{l}\mathrm{Fe}_{2} \mathrm{O}_{3} \\
\text { (total) }\end{array}$ & . & 12.88 & 0.53 & 4.1 & 12.35 & 13.41 & 13.85 & - & . & 12.0 & 14.5 & +0.97 & 7.5 & 9.67 & 9.73 & 0.29 & 3.0 & 9.44 & 10.02 & 9.34 & 0.54 & 5.8 & 8.79 & 9.88 & -0.33 & 3.4 \\
\hline
\end{tabular}

Note: $R . V .=$ recommended values; $\mathrm{X}=$ average values; $\mathrm{S}=$ stand ard deviation; $\mathrm{C}=$ relative deviation. 


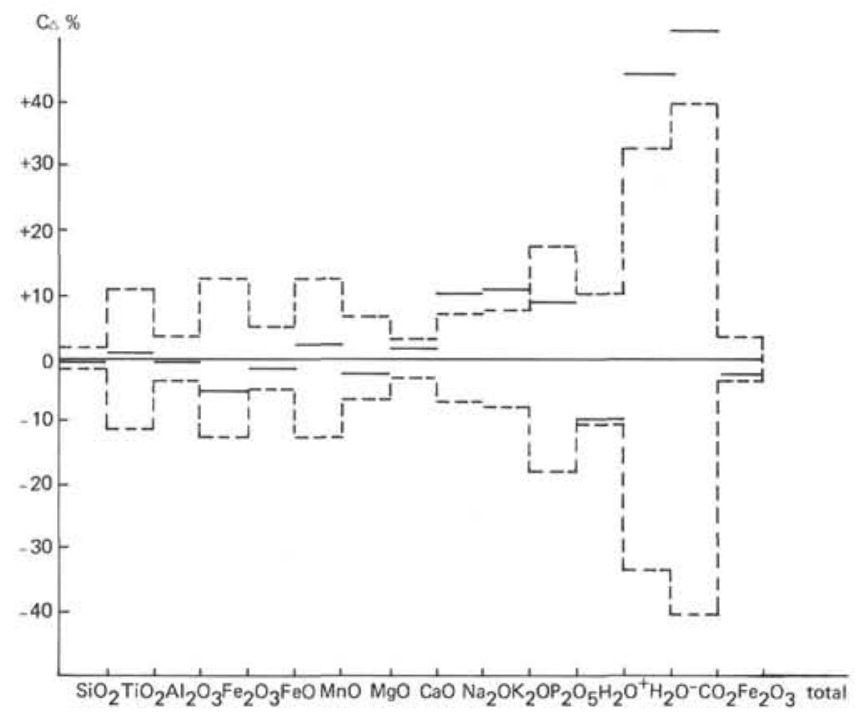

Figure 2. Intervals of relative deviations for diorite $D R-N$. Symbols as in Figure 1.

than could be obtained if the rocks were powdered under vacuum.

The data given on the chemical compositions and amounts of gases in the samples should be regarded as qualitative. A group of low-boiling gases $\left(\mathrm{H}_{2}, \mathrm{O}_{2}, \mathrm{~N}_{2}, \mathrm{CO}_{2}\right.$, and $\left.\mathrm{CH}_{4}\right)$ was analyzed by means of a standard chromatograph LKHM-8D equipped with a catarometer. For determination of hydrocarbon gases up to $\mathrm{C}_{5}$, including isomers $\mathrm{C}_{4}-\mathrm{C}_{5}$ and olefins $\mathrm{C}_{2}-\mathrm{C}_{4}$, we used the chromatograph "Geokhimik" equipped with a hydrogen flame ionization detector.

\section{RESULTS AND DISCUSSION \\ Petrochemistry of Basalts}

Petrographic study of basalts revealed that aphyric varieties are predominant and formed, as a rule, a series of flows overlapping one another. Among rare porphyritic basalts, the olivine-plagioclase microphyric varieties are the most common, whereas the plagioclase porphyritic basalts occur sporadically. All varieties of basalts were analyzed, and 85 complete silicate analyses (Table 2) were conducted. The differences in the chemical composition of the varieties of basalts are insignificant. Greater chemical differences occur, however, among basalts developed in various parts of the mid-oceanic ridge. Thus, all analyses were organized into three groups according to sample location.

The first group includes analyses of the Reykjanes Ridge at $62^{\circ} \mathrm{N}$ (Holes 407, 408, and 409). The second group of samples is from the Mid-Atlantic Ridge at $45^{\circ} \mathrm{N}$ (Holes 410 and $410 \mathrm{~A})$. The third group consists of samples from the MidAtlantic Ridge at $36^{\circ} \mathrm{N}$ (Holes 411, 412A, and 413).

Distribution histograms of the major-forming elements elucidate an important peculiarity of the basalt chemistry: every element is characterized by various modal values in each of the groups concerned. Some elements in basalts from the Reykjanes Ridge show bimodal (aluminum, calcium) and even three-modal (titanium) distributions (Figure 3). This peculiarity is interpreted as the result of differences in the composition of primary basaltic melts occurring in various parts of the Mid-Atlantic Ridge.
In the bicomponent variation diagram for $\mathrm{Al}_{2} \mathrm{O}_{3}-\mathrm{TiO}_{2}$ (Figure 4) the ratio values are grouped in three fields and indicate pronounced reverse correlative dependence of these two components. The first field describes the $\mathrm{Al}_{2} \mathrm{O}_{3} / \mathrm{TiO}_{2}$ ratio in basalts from the Reykjanes Ridge. The diagram shows that the younger the basalt the higher the $\mathrm{Al}_{2} \mathrm{O}_{3}$ concentrations and the lower the $\mathrm{TiO}_{2}$ contents. In basalts from Hole 409, the ratios are the same as in abyssal tholeiites and similar to the ratios in basalts from the remaining holes of Leg 49 (Figure 4, fields 2 and 3). Basalts from Hole 407 are characterized by anomalously high $\mathrm{TiO}_{2}$ contents with low concentrations of $\mathrm{Al}_{2} \mathrm{O}_{3}$.

In the variation diagrams for $\mathrm{Al}_{2} \mathrm{O}_{3}-\mathrm{MgO}$ (Figure 5), $\mathrm{Al}_{2} \mathrm{O}_{3}-\mathrm{CaO}$ (Figure 6), and $\mathrm{CaO}-\mathrm{MgO}$ (Figure 7), one can see that the fields of values of basalts from various parts of the Mid-Atlantic Ridge partly overlap one another. On the basis of these components, basalts from Hole 407 (the oldest among the rocks studied) differ considerably from those of other holes. Basalts from Hole 409 (the youngest in the Reykjanes Ridge) are similar to abyssal tholeiites.

The distribution of $\mathrm{K}_{2} \mathrm{O}$ contents was unexpected. First, an extremely wide interval of concentrations $(0.14$ to 2.41 wt.\%) occurs and very high $\mathrm{K}_{2} \mathrm{O}$ content are peculiar to Hole 410. In the variation diagram for $\mathrm{K}_{2} \mathrm{O}-\mathrm{TiO}_{2}$ (Figure 8), the basalts developed in various regions of the Mid-Atlantic Ridge are characterized by various correlative ratios between these two components. A weak correlative dependence between $\mathrm{K}_{2} \mathrm{O}$ and $\mathrm{TiO}_{2}$ characterizes basalts of the Reykjanes Ridge; a stronger dependence typifies basalts of the MidAtlantic Ridge at $36^{\circ} \mathrm{N}$. Basalts of the Mid-Atlantic Ridge at $45^{\circ} \mathrm{N}$ are characterized by weak reverse correlative dependence between $\mathrm{K}_{2} \mathrm{O}$ and $\mathrm{TiO}_{2}$. In this diagram, and in previous ones, the fields overlap one another within the diagram in a manner characteristic of abyssal tholeiites. Basalts from Hole 409 of the Reykjanes Ridge are most similar to tholeiites, whereas basalts from Holes 407 and 410 are different from tholeiites.

The variation diagram $\mathrm{TiO}_{2}-\mathrm{F} / \mathrm{M}$ (where $\mathrm{F}$ is the total $\mathrm{Fe}$ content recalculated to $\mathrm{FeO}, \mathrm{M}$ represents $\mathrm{MgO}$ ) shows that basalts of the Mid-Atlantic Ridge are characterized by a strong direct correlative dependence between $\mathrm{TiO}_{2}$ content and the F/M ratio (Figure 9). The ratios for basalts from Holes 409, 410, and 412A are similar to those of abyssal tholeiites. Basalts from Hole 407 and part of Hole 408 differ considerably from abyssal tholeiites, according to this characteristic.

The Gottini (1970) coefficient $(t)$ and that of $\mathrm{TiO}_{2}$ are associated by parabolic dependence (Figure 10). Basalts from all holes, except Hole 407, occupy a position in the Gottini diagram peculiar to abyssal tholeiites.

In the Miyashiro (1975) variation diagram $\mathrm{Na}_{2} \mathrm{O} / \mathrm{K}_{2} \mathrm{O}$ $\left(\mathrm{Na}_{2} \mathrm{O}+\mathrm{K}_{2} \mathrm{O}\right)$, the Mid-Atlantic Ridge basalts plot very compactly (Figure 10). They lie almost entirely within the field of Icelandic tholeiites, a portion of which falls within the field of abyssal tholeiites, and the remaining part within the field of alkaline basalts of the Atlantic islands. The alkaline tendency is characteristic generally for basalts from Holes 410 and 410A (Figure 11). Noteworthy is that in this diagram all the basalts studied plot beneath the boundary line $\mathrm{V}-\mathrm{V}$ separating the fresh rocks from those altered by epimagmatic processes. 

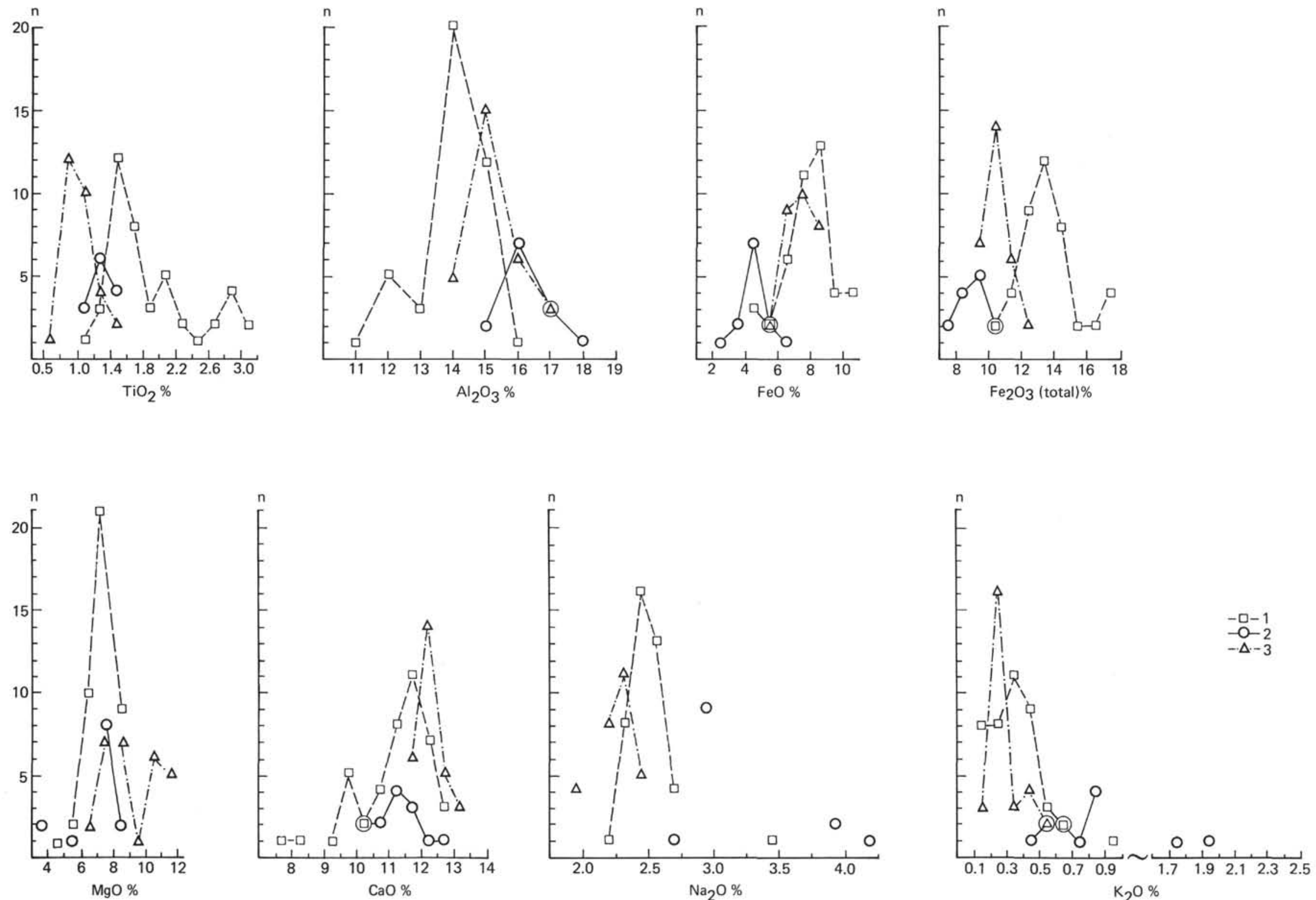

Figure 3. Histogram of major-element distribution. $1=$ basalts from Holes 407, 408, and 409; $2=$ basalts from Holes 410 and $410 \mathrm{~A} ; 3=$ basalts from Holes 411 , $412 A$, and $413 ; n$ - number of determinations. 


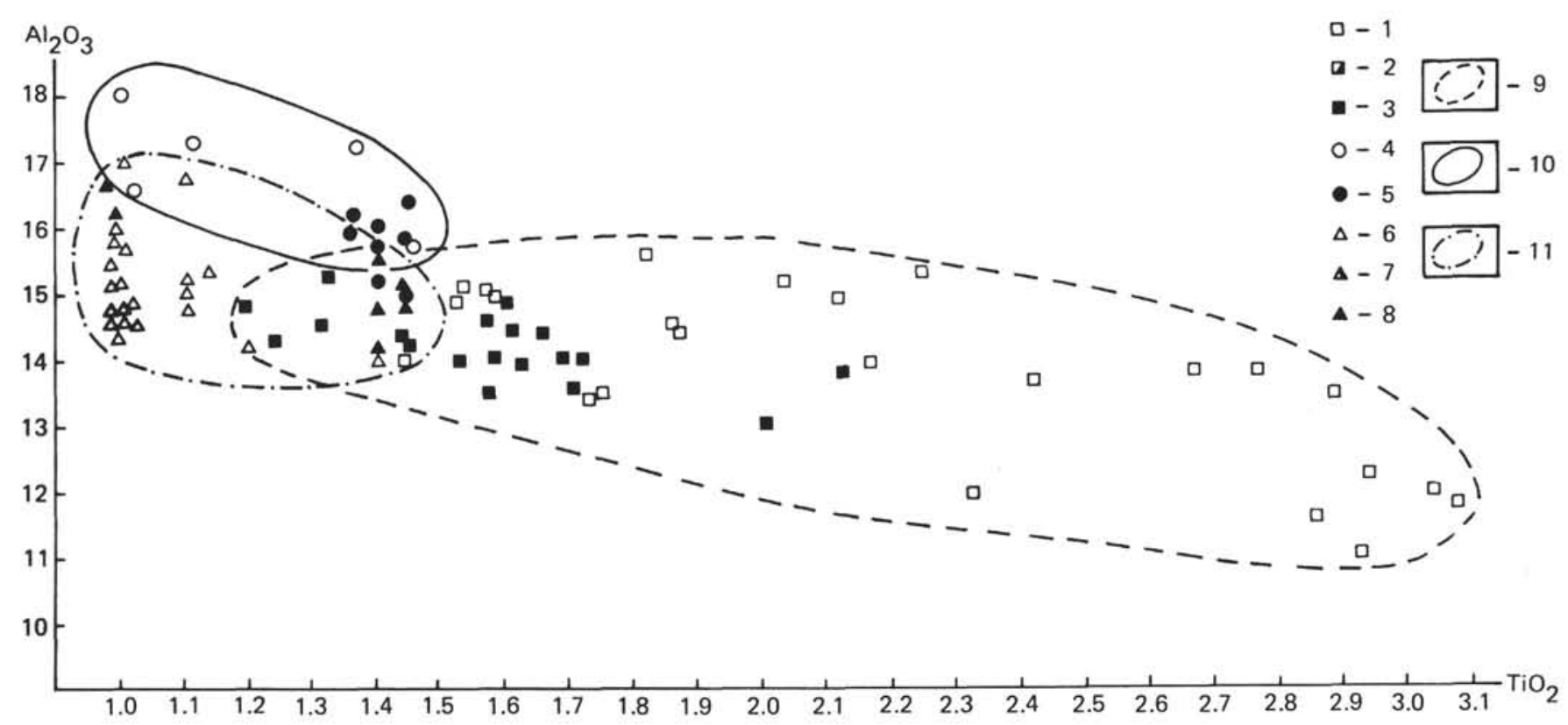

Figure 4. Variation diagram $\mathrm{Al}_{2} \mathrm{O}_{3}-\mathrm{TiO}_{2}$. Holes: 1 - 407, 2 - 408, 3- 409, 4-410, 5-410A, 6-411, 7-412A, 8- 413. Regions: 9 - the Reykjanes Ridge, 10 - the Mid-Atlantic Ridge at $45^{\circ} \mathrm{N}, 11$ - the Mid-Atlantic Ridge at $36^{\circ} \mathrm{N}$.

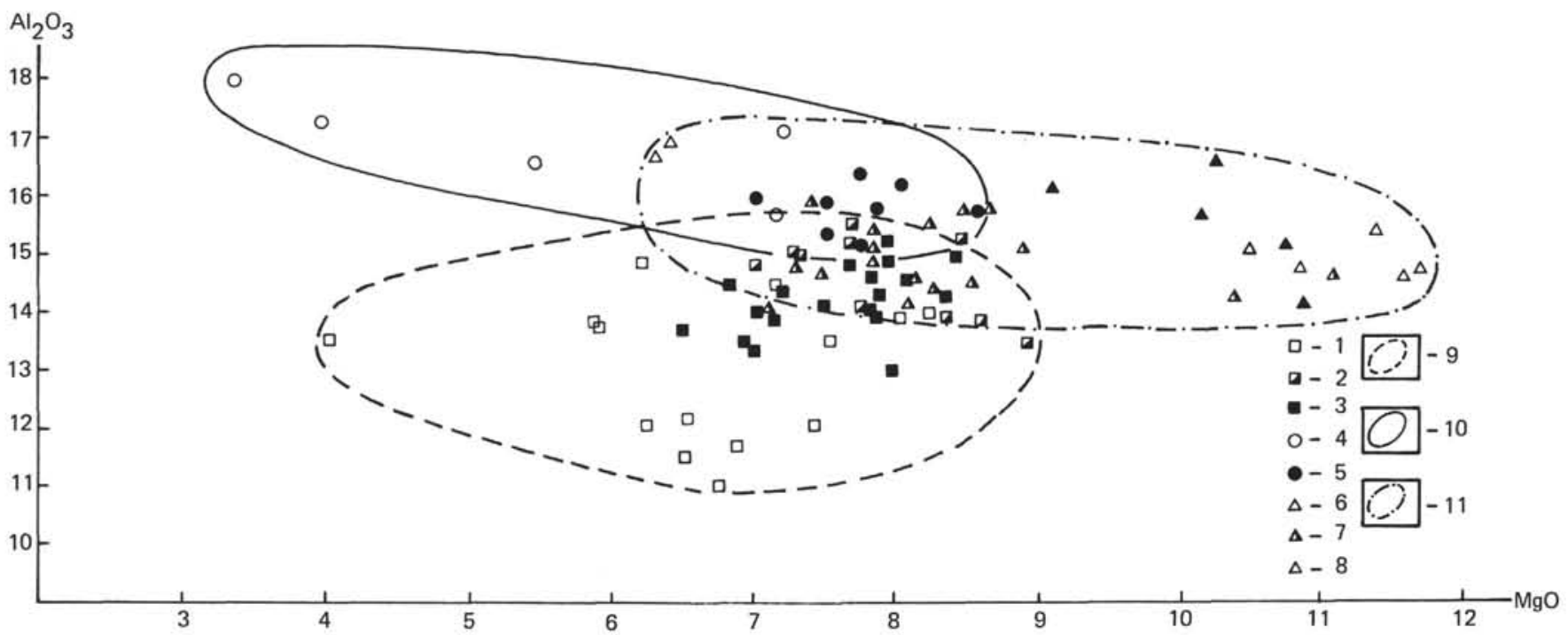

Figure 5. Variation diagram $\mathrm{Al}_{2} \mathrm{O}_{3}-\mathrm{MgO}$. Symbols as in Figure 4.

Basalts of the mid-oceanic ridges are believed to be attributable to a series of abyssal tholeiites, but basalts penetrated during Leg 49 can be subdivided into three series according to Kuno (1966) classification. Holes 408, 409, 411,412 , and $412 \mathrm{~A}$ shown in the three-component AFM diagram (Figure 12), fall within the field of the highly aluminous series. Basalts from Hole 407 occur within the field of the tholeiitic series. These two series form a single evolutionary row characterized by the Fenner trend of differentiation. Basalts from Holes 410 and $410 \mathrm{~A}$ belong to the alkaline olivine-basaltic series, and are characterized by the Bowen differentiation trend (Figure 12).

Similar conclusions can be drawn after examining the diagram compiled by de La Roche and Letterier (1973). In this diagram (Figure 13), representing a chemical modification of the normative tetrahedron of Yoder and Tilly (1962), the basalts of the Mid-Atlantic Ridge also fall within three fields corresponding to different series: tholeiitic, highly aluminous, and alkaline-olivine-basaltic. A greater part of the basalts studied belong to the highly aluminous series, when judged by composition. In this case, rocks from Hole 407 exhibit a barely observable tendency towards a rhyolitic type of differentiation, whereas basalts from Hole 410 tend to the trachytic type (Figure 13).

The examination of petrochemical diagrams shows that basalts of the Mid-Atlantic Ridge penetrated during Leg 49 are slightly differentiated after $\mathrm{SiO}_{2}$. According to classifications by Kuno (1966) and de La Roche (1973), they can be divided into three series: tholeiitic, high-alumina, and alkaline-olivine-basaltic, with the high alumina series in its quantitative ratio. We can conclude that there is a lateral geochemical heterogeneity of different ages of basalts that 
TABLE 2

Chemical Composition of Magmatic Rocks From Holes 407, 408, 409, 410, 410A, 411, 412A, and 413, Leg 49

\begin{tabular}{|c|c|c|c|c|c|c|c|c|c|c|c|c|c|c|}
\hline Hole & & & & & & & & 407 & & & & & & \\
\hline Core & 35 & 36 & 36 & 37 & 37 & 38 & 39 & 39 & 40 & 42 & 44 & 45 & 46 & 47 \\
\hline Section & 1 & 1 & 3 & 1 & 2 & 2 & 2 & 3 & 1 & 2 & 1 & 1 & 2 & 3 \\
\hline Interval $(\mathrm{cm})$ & $30-33$ & $20-25$ & $95-97$ & $112-117$ & $109-111$ & $15-17$ & $100-105$ & $25-30$ & $70-75$ & $35-40$ & $60-65$ & $55-60$ & $0-5$ & $77-80$ \\
\hline $\mathrm{SiO}_{2}$ & 53.91 & 47.67 & 47.35 & 47.15 & 47.66 & 47.95 & 47.78 & 48.09 & 48.07 & 48.37 & 46.27 & 45.88 & 46.25 & 47.20 \\
\hline $\mathrm{TiO}_{2}$ & 2.88 & 1.73 & 1.75 & 3.07 & 2.92 & 2.94 & 2.85 & 2.76 & 3.04 & 2.66 & 2.12 & 2.32 & 1.86 & 1.87 \\
\hline $\mathrm{Al}_{2} \mathrm{O}_{3}$ & 13.59 & 13.98 & 13.50 & 11.78 & 11.09 & 12.24 & 11.57 & 13.85 & 12.25 & 13.88 & 14.96 & 12.04 & 14.58 & 14.04 \\
\hline $\mathrm{Fe}_{2} \mathrm{O}_{3}$ & 4.91 & 7.41 & 5.95 & 7.23 & 6.42 & 4.89 & 6.55 & 3.75 & 5.71 & 4.89 & 6.16 & 9.20 & 6.99 & 4.59 \\
\hline $\mathrm{FeO}$ & 7.31 & 5.77 & 8.14 & 9.13 & 10.31 & 10.23 & 10.30 & 9.94 & 10.37 & 8.72 & 6.69 & 6.21 & 6.49 & 7.93 \\
\hline $\mathrm{MnO}$ & 0.15 & 0.14 & 0.17 & 0.20 & 0.23 & 0.24 & 0.25 & 0.18 & 0.24 & 0.21 & 0.18 & 0.20 & 0.17 & 0.17 \\
\hline $\mathrm{MgO}$ & 4.04 & 8.02 & 7.53 & 6.85 & 6.72 & 6.53 & 6.53 & 5.92 & 6.25 & 5.89 & 6.24 & 7.41 & 7.17 & 8.22 \\
\hline $\mathrm{CaO}$ & 7.57 & 10.58 & 11.70 & 10.32 & 10.28 & 10.00 & 9.88 & 11.12 & 9.90 & 11.09 & 12.12 & 10.52 & 11.43 & 11.18 \\
\hline $\mathrm{Na}_{2} \mathrm{O}$ & 3.49 & 2.70 & 2.41 & 2.61 & 2.61 & 2.61 & 2.61 & 2.56 & 2.51 & 2.42 & 2.49 & 2.70 & 2.38 & 2.28 \\
\hline $\mathrm{K}_{2} \mathrm{O}$ & 0.99 & 0.31 & 0.20 & 0.34 & 0.41 & 0.54 & 0.61 & 0.39 & 0.58 & 0.46 & 0.28 & 0.61 & 0.28 & 0.15 \\
\hline $\mathrm{H}_{2} \mathrm{O}^{+}$ & 0.63 & 0.80 & 0.35 & 0.43 & 0.68 & 0.58 & 0.79 & 0.64 & 0.53 & 0.49 & 0.91 & 1.20 & 1.25 & 1.19 \\
\hline $\mathrm{H}_{2} \mathrm{O}^{-}$ & 0.49 & 0.97 & 0.73 & 0.86 & 0.59 & 0.56 & 0.42 & 0.84 & 0.47 & 0.69 & 1.23 & 1.58 & 1.09 & 1.36 \\
\hline $\mathrm{P}_{2} \mathrm{O}_{5}$ & 0.52 & 0.14 & 0.13 & 0.26 & 0.26 & 0.30 & 0.29 & 0.26 & 0.29 & 0.27 & 0.27 & 0.25 & 0.19 & 0.19 \\
\hline $\mathrm{CO}_{2}$ & $\cdot$ & 0.20 & - & 0.30 & 0.10 & - & 0.15 & - & 0.25 & $\cdot$ & $\therefore$ & 0.20 & . & - \\
\hline Total & 100.48 & 100.42 & 99.91 & 100.53 & 100.28 & 99.61 & 100.58 & 100.30 & 100.46 & 100.04 & 99.92 & 100.32 & 100.03 & 100.37 \\
\hline $\mathrm{T}$ & 3.51 & 6.52 & 6.34 & 2.99 & 2.9 & 3.28 & 3.14 & 4.09 & 3.2 & 4.32 & 5.9 & 4.08 & 6.61 & 6.31 \\
\hline Y & 1268 & 1756 & 1890 & 1670 & 1660 & 1630 & 1610 & 1760 & 1610 & 1650 & 1840 & 1720 & 1860 & 1870 \\
\hline $\mathrm{X}$ & 17.64 & 1886 & 1834 & 1640 & 1640 & 1690 & 1600 & 1800 & 1700 & 1840 & 1750 & 1590 & 1850 & 1970 \\
\hline A & 22.12 & 12.86 & 11.04 & 11.06 & 11.7 & 13.0 & 12.4 & 13.3 & 12.4 & 13.2 & 13.1 & 13.1 & 11.3 & 10.7 \\
\hline $\mathrm{F}$ & 57.93 & 53.00 & 57.10 & 61.2 & 62.4 & 60.1 & 62.5 & 60.2 & 62.2 & 59.9 & 57.5 & 57.5 & 56.6 & 53.3 \\
\hline M & 19.95 & 34.27 & 31.86 & 26.3 & 26.0 & 26.9 & 25.1 & 26.5 & 25.4 & 26.9 & 29.4 & 29.4 & 32.1 & 36.0 \\
\hline
\end{tabular}

\begin{tabular}{|c|c|c|c|c|c|c|c|c|c|c|c|c|c|c|}
\hline \multicolumn{15}{|l|}{ Hole } \\
\hline Core & 15 & 17 & 18 & 20 & 21 & 24 & 24 & 25 & 25 & 28 & 31 & 31 & 32 & 37 \\
\hline Section & 2 & 1 & 1 & 1 & 3 & 2 & 3 & 2 & 4 & 1 & 1 & 2 & 1 & 1 \\
\hline Interval $(\mathrm{cm})$ & $80-85$ & $130-135$ & $90-95$ & $10-15$ & $120-125$ & $130-135$ & $145-150$ & $60-65$ & $40-45$ & $120-125$ & $70-75$ & $45-48$ & $85-98$ & $85-90$ \\
\hline $\mathrm{SiO}_{2}$ & 47.17 & 47.37 & 47.15 & 47.11 & 47.83 & 48.82 & 48.16 & 47.87 & 47.27 & 47.34 & 47.56 & 47.73 & 48.57 & 50.48 \\
\hline $\mathrm{TiO}_{2}$ & 1.53 & 1.57 & 1.65 & 2.07 & 1.57 & 1.44 & 1.69 & 2.12 & 1.70 & 1.62 & 1.58 & 1.61 & 1.57 & 1.45 \\
\hline $\mathrm{Al}_{2} \mathrm{O}_{3}$ & 13.99 & 15.04 & 14.38 & 13.02 & 14.66 & 14.41 & 14.01 & 13.79 & 13.59 & 13.91 & 14.07 & 14.43 & 13.50 & 15.77 \\
\hline $\mathrm{Fe}_{2} \mathrm{O}_{3}$ & 5.21 & 2.75 & 4.85 & 6.39 & 3.39 & 4.15 & 3.50 & 4.98 & 6.33 & 5.90 & 5.34 & 4.82 & 4.91 & 2.15 \\
\hline $\mathrm{FeO}$ & 7.55 & 8.61 & 8.15 & 8.12 & 8.38 & 8.38 & 9.20 & 9.32 & 7.27 & 7.16 & 7.38 & 7.17 & 8.06 & 6.18 \\
\hline Mno & 0.19 & 0.19 & 0.19 & 0.21 & 0.17 & 0.20 & 0.17 & 0.21 & 0.20 & 0.18 & 0.18 & 0.24 & 0.20 & 0.16 \\
\hline $\mathrm{MgO}$ & 7.12 & 8.43 & 7.89 & 7.97 & 7.82 & 7.17 & 7.81 & 6.49 & 6.94 & 7.84 & 7.06 & 6.82 & 6.95 & 7.15 \\
\hline $\mathrm{CaO}$ & 12.49 & 11.98 & 11.85 & 10.74 & 11.98 & 12.47 & 11.59 & 12.00 & 12.02 & 11.20 & 12.08 & 12.00 & 11.58 & 11.22 \\
\hline $\mathrm{Na}_{2} \mathrm{O}$ & 2.55 & 2,46 & 2.31 & 2.32 & 2.43 & 2.43 & 2.43 & 2.62 & 2.52 & 2.33 & 2.48 & 2.46 & 2.25 & 2.88 \\
\hline $\mathrm{K}_{2} \mathrm{O}$ & 0.43 & 0.20 & 0.33 & 0.24 & 0.31 & 0.27 & 0.22 & 0.39 & 0.39 & 0.46 & 0.35 & 0.37 & 0.37 & 0.83 \\
\hline $\mathrm{H}_{2}^{-} \mathrm{O}^{+}$ & 0.61 & 0.24 & 0.59 & 0.61 & 0.72 & 0.32 & 0.66 & 0.26 & 0.66 & 0.71 & 0.68 & 0.58 & 0.57 & 0.79 \\
\hline $\mathrm{H}_{2} \mathrm{O}^{-}$ & 0.64 & 0.56 & 0.70 & 0.74 & 0.62 & 0.16 & 0.48 & 0.39 & 0.94 & 1.34 & 0.97 & 1.16 & 0.95 & 0.48 \\
\hline $\mathrm{P}_{2} \mathrm{O}_{5}$ & 0.18 & 0.18 & 0.14 & 0.19 & 0.15 & 0.18 & 0.16 & 0.25 & 0.18 & 0.13 & 0.17 & 0.16 & 0.14 & 0.22 \\
\hline $\mathrm{CO}_{2}$ & 0.45 & - & - & - & - & - & 0.10 & - & 0.40 & 0.05 & 0.25 & - & $\cdot$ & - \\
\hline Total & 100.11 & 99.58 & 100.18 & 99.73 & 100.03 & 100.40 & 100.18 & 100.68 & 100.41 & 100.17 & 100.15 & 99.55 & 99.62 & 99.76 \\
\hline $\mathrm{T}$ & 7.48 & 8.01 & 7.82 & 5.17 & 7.77 & 8.33 & 6.86 & 5.27 & 6.51 & 7.15 & 7.34 & 7.75 & 7.2 & 8.9 \\
\hline$Y$ & 1950 & 1982 & 1928 & 1800 & 1940 & 1970 & 1880 & 1870 & 1890 & 1870 & 1890 & 1900 & 1830 & 1870 \\
\hline $\mathrm{X}$ & 1780 & 1900 & 1870 & 1850 & 1900 & 1970 & 1910 & 1760 & 1800 & 1860 & 1870 & 1900 & 2010 & 1890 \\
\hline A & 13.3 & 11.0 & 11.4 & 10.5 & 12.2 & 12.3 & 11.6 & 12.9 & 12.8 & 12.1 & 12.8 & 13.4 & 11.9 & 19.5 \\
\hline $\mathrm{F}$ & 54.8 & 50.0 & 54.4 & 56.7 & 52.1 & 55.0 & 54.1 & 59.2 & 56.8 & 54.0 & 55.3 & 54.5 & 56.7 & 42.7 \\
\hline $\mathrm{M}$ & 31.9 & 39.0 & 34.2 & 32.8 & 35.7 & 32.7 & 34.3 & 27.9 & 30.4 & 53.9 & 31.9 & 32.1 & 31.4 & 37.8 \\
\hline
\end{tabular}

\begin{tabular}{|c|c|c|c|c|c|c|c|c|c|c|c|c|c|c|}
\hline \multicolumn{15}{|l|}{ Hole } \\
\hline Core & 3 & 4 & 1 & 2 & 3 & 3 & 3 & 5 & 6 & 7 & 8 & 9 & 11 & 11 \\
\hline Section & 1 & 1 & 1 & 1 & 1 & 2 & 3 & 1 & 1 & 2 & 1 & 1 & 2 & 3 \\
\hline Interval $(\mathrm{cm})$ & $143-145$ & $84-88$ & $16-20$ & $71-75$ & $47-50$ & $45-50$ & $42-45$ & $35-40$ & $85-90$ & $130-135$ & $90-95$ & $130-135$ & $140-145$ & $3-5$ \\
\hline $\mathrm{SiO}_{2}$ & 49.76 & 49.81 & 49.79 & 49.19 & 49.95 & 49.70 & 49.11 & 49.77 & 49.70 & 50.45 & 49.82 & 50.51 & 49.70 & 50.06 \\
\hline $\mathrm{TiO}_{2}$ & 1.10 & 1.02 & 1.19 & 0.98 & 1.02 & 0.93 & 1.40 & 1.02 & 1.10 & 0.99 & 0.98 & 1.10 & 0.89 & 0.94 \\
\hline $\mathrm{Al}_{2} \mathrm{O}_{3}$ & 16.70 & 16.98 & 14.12 & 15.92 & 14.66 & 14.30 & 14.07 & 14.45 & 14.86 & 14.69 & 14.69 & 15.19 & 15.14 & 15.83 \\
\hline $\mathrm{Fe}_{2} \mathrm{O}_{3}$ & 3.16 & 2.71 & 2.62 & 3.43 & 2.50 & 1.93 & 3.20 & 2.97 & 3.57 & 3.02 & 1.87 & 1.48 & 1.89 & 1.96 \\
\hline $\mathrm{FeO}$ & 6.41 & 6.21 & 7.97 & 6.17 & 7.79 & 7.66 & 8.56 & 8.31 & 7.04 & 6.98 & 8.21 & 8.10 & 7.40 & 6.87 \\
\hline $\mathrm{MnO}$ & 0.15 & 0.15 & 0.16 & 0.15 & 0.17 & 0.15 & 0.20 & 0.17 & 0.18 & 0.17 & 0.16 & 0.17 & 0.15 & 0.14 \\
\hline $\mathrm{MgO}$ & 6.32 & 6.41 & 8.08 & 7.47 & 8.10 & 10.38 & 7.11 & 8.28 & 7.27 & 7.46 & 8.56 & 7.84 & 8.91 & 8.64 \\
\hline $\mathrm{CaO}$ & 13.26 & 13.29 & 12.44 & 12.75 & 12.37 & 11.98 & 12.24 & 12.26 & 12.79 & 12.95 & 12.50 & 12.39 & 12.86 & 12.86 \\
\hline $\mathrm{Na}_{2} \mathrm{O}$ & 2.34 & 2.25 & 2.34 & 2.31 & 2.31 & 2.15 & 2.39 & 2.39 & 2.25 & 2.31 & 2.15 & 2.33 & 2.23 & 2.33 \\
\hline $\mathrm{K}_{2} \mathrm{O}$ & 0.41 & 0.37 & 0.29 & 0.28 & 0.28 & 0.27 & 0.27 & 0.27 & 0.56 & 0.33 & 0.27 & 0.26 & 0.20 & 0.26 \\
\hline $\mathrm{H}_{2} \mathrm{O}^{+}$ & 0.39 & 0.36 & 0.36 & 0.38 & 0.43 & 0.25 & 0.45 & 0.31 & 0.52 & 0.25 & 0.15 & 0.45 & 0.30 & 0.24 \\
\hline $\mathrm{H}_{2} \mathrm{O}^{-}$ & 0.10 & 0.12 & 0.24 & 0.52 & 0.32 & 0.30 & 0.40 & 0.34 & 0.28 & 0.24 & 0.50 & 0.24 & 0.30 & 0.36 \\
\hline $\mathrm{P}_{2} \mathrm{O}_{5}$ & 0.16 & 0.11 & 0.07 & 0.10 & 0.10 & 0.10 & 0.11 & 0.10 & 0.14 & 0.10 & 0.10 & 0.10 & 0.08 & 0.07 \\
\hline $\mathrm{CO}_{2}$ & . & $\cdot$ & $\because$ & 0.60 & - & - & - & - & 0.05 & 0.10 & $\because$ & - & - & $\cdot$ \\
\hline Total & 100.27 & 99.79 & 99.67 & 100.19 & 100.00 & 100.10 & 99.51 & 100.64 & 100.31 & 100.04 & 99.90 & 100.16 & 100.05 & 100.56 \\
\hline $\mathrm{T}$ & 13.1 & 14.4 & 9.92 & 13.9 & 12.2 & 13.1 & 8.36 & 11.9 & 11.5 & 12.5 & 12.8 & 11.7 & 14.5 & 14.4 \\
\hline $\mathrm{Y}$ & 2060 & 2060 & 2010 & 2030 & 2000 & 2060 & 1940 & 2000 & 2010 & 2030 & 2050 & 1990 & 2110 & 2110 \\
\hline $\mathrm{X}$ & 2120 & 2220 & 2140 & 2140 & 2160 & 2240 & 2060 & 2120 & 2120 & 2190 & 2230 & 2210 & 2230 & 2210 \\
\hline A & 14.9 & 14.9 & 12.5 & 13.6 & 12.6 & 10.9 & 12.5 & 12.1 & 13.8 & 13.3 & 11.6 & 13.1 & 11.9 & 13.0 \\
\hline $\mathrm{F}$ & 50.3 & 48.9 & 49.0 & 48.0 & 48.3 & 42.3 & 53.8 & 50.0 & 50.5 & 49.0 & 47.3 & 47.6 & 44.6 & 43.4 \\
\hline M & 34.8 & 36.2 & 38.5 & 38.4 & 39.1 & 46.8 & 33.7 & 37.9 & 35.7 & 37.7 & 41.1 & 39.3 & 43.5 & 43.5 \\
\hline
\end{tabular}

Note: $\mathrm{T}=\frac{\mathrm{Al}_{2} \mathrm{O}_{3}-\mathrm{Na}_{2} \mathrm{O}}{\mathrm{TiO}_{2}} ; \mathrm{y}=6 \mathrm{Ca}+2 \mathrm{Mg}+\mathrm{Al} ; \mathrm{X}=4 \mathrm{Si}-11(\mathrm{Na}+\mathrm{K})-2(\mathrm{Fe}+\mathrm{Ti}) ; \mathrm{A}=\mathrm{Na}_{2} \mathrm{O}+\mathrm{K}_{2} \mathrm{O} ; \mathrm{F}=\mathrm{Fe}_{2} \mathrm{O}_{3} \cdot 0.9+\mathrm{FeO} ; \mathrm{M}=\mathrm{MgO} ;$ Indices $\mathrm{A} . \mathrm{F} . \mathrm{M}$. scaled to $100 \%$. 
TABLE 2 - Continued

\begin{tabular}{|c|c|c|c|c|c|c|c|c|c|c|c|c|c|c|c|}
\hline 36 & 36 & 36 & 36 & 37 & 37 & 37 & 38 & 38 & 38 & 7 & 9 & 10 & 10 & 11 & 12 \\
\hline 1 & 2 & 3 & 5 & 1 & 2 & 3 & 1 & 2 & 3 & 6 & 2 & 1 & 4 & 3 & 1 \\
\hline $121-124$ & $83-85$ & $7 \cdot 10$ & $34-37$ & $74-77$ & 89.91 & $33-35$ & $66-68$ & $38-40$ & $95-97$ & $135-140$ & $95-100$ & $50-55$ & $95-100$ & $70-75$ & 120.125 \\
\hline 45.85 & 47.08 & 46.76 & 47.24 & 48.61 & 46.54 & 47.05 & 45.93 & 45.81 & 45.90 & 48.61 & 49.99 & 49.21 & 49.17 & 49,19 & 48.86 \\
\hline 1.71 & 1.53 & 1.52 & 1.57 & 1.44 & 2.03 & 1.82 & 2.41 & 2.16 & 2.24 & 1.60 & 1.45 & 1.32 & 1.31 & 1.23 & 1.19 \\
\hline 14.07 & 15.07 & 14.89 & 15.04 & 14.01 & 15.21 & 15.61 & 13.67 & 13.90 & 15.32 & 14.90 & 14.23 & 15.30 & 14.54 & 14.37 & 14.87 \\
\hline 7.60 & 5.06 & 6.01 & 6.15 & 3.11 & 5.36 & 4.03 & 5.88 & 6.68 & 4.77 & 5.11 & 3.51 & 1.90 & 2.95 & 2.73 & 3.39 \\
\hline 5.29 & 4.85 & 4.90 & 4.71 & 7.05 & 6.45 & 6.87 & 7.83 & 7.06 & 6.79 & 8.13 & 8.01 & 8.53 & 8.46 & 8.61 & 7.77 \\
\hline 0.15 & 0.11 & 0.14 & 0.13 & 0.11 & 0.09 & 0.16 & 0.18 & 0.21 & 0.19 & 0.17 & 0.20 & 0.17 & 0.17 & 0.17 & 0.18 \\
\hline 7.78 & 7.32 & 7.03 & 7.30 & 8.37 & 7.68 & 7.69 & 8.92 & 8.59 & 8.46 & 7.67 & 7.49 & 7.94 & 8.08 & 8.37 & 7.92 \\
\hline 11.28 & 12.58 & 12.55 & 11.96 & 11.60 & 9.77 & 11.36 & 8.16 & 9.12 & 9.68 & 10.86 & 11.08 & 12.27 & 11.85 & 12.14 & 12.74 \\
\hline 2.51 & 2.42 & 2.35 & 2.49 & 2.35 & 2.70 & 2.49 & 2.70 & 2.41 & 2.51 & 2.41 & 2.51 & 2.55 & 2.31 & 2.39 & 2.39 \\
\hline 0.58 & 0.41 & 0.48 & 0.48 & 0.26 & 0.41 & 0.20 & 0.41 & 0.24 & 0.24 & 0.17 & 0.37 & 0.14 & 0.16 & 0.20 & 0.20 \\
\hline 0.84 & 1.17 & 1.26 & 1.12 & 0.90 & 1.48 & 1.31 & 1.62 & 1.57 & 1.71 & 0.67 & 0.44 & 0.52 & 0.43 & 0.43 & 0.39 \\
\hline 1.54 & 1.77 & 1.70 & 1.62 & 1.02 & 2.03 & 1.45 & 1.64 & 2.02 & 1.57 & 0.45 & 0.22 & 0.32 & 0.52 & 0.42 & 0.36 \\
\hline 0.17 & 0.14 & 0.13 & 0.18 & 0.11 & 0.23 & 0.18 & 0.29 & 0.24 & 0.24 & 0.11 & 0.14 & 0.10 & 0.09 & 0.09 & 0.08 \\
\hline 0.45 & 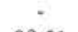 & $\cdot$ & 0.05 & $\cdot$ & $\cdot$ & 0.10 & - & - & - & . & $\therefore$ & $\cdot$ & - & - & - \\
\hline 99.83 & 99.51 & 99.72 & 100.12 & 99.84 & 100.03 & 100.32 & 99.64 & 100.01 & 99.62 & 100.13 & 99.64 & 100.27 & 100.04 & 100.34 & 100.34 \\
\hline 6.74 & 8.3 & 8.22 & 8.03 & 8.13 & 6.16 & 7.2 & 4.56 & 5.32 & 5.71 & 7.81 & 8.07 & 9.7 & 9.31 & 9.79 & 10.5 \\
\hline 1850 & 1990 & 1970 & 1920 & 1930 & 1720 & 1900 & 1580 & 1660 & 1750 & 1830 & 1820 & 2010 & 1940 & 2000 & 2050 \\
\hline 1690 & 1890 & 1890 & 1860 & 2080 & 1680 & 1830 & 1580 & 1740 & 1770 & 1950 & 2040 & 2060 & 2100 & 2060 & 2070 \\
\hline 13.4 & 14.5 & 14.1 & 14.4 & 12.4 & 14.1 & 12.9 & 12.4 & 10.9 & 12.3 & 11.2 & 13.3 & 12.9 & 11.4 & 11.8 & 12.2 \\
\hline 52.6 & 48.2 & 51.7 & 50.0 & 47.4 & 51.1 & 50.3 & 52.1 & 53.7 & 49.9 & 55.5 & 51.9 & 49.0 & 51.2 & 50.3 & 50.7 \\
\hline 34.0 & 37.3 & 34.2 & 35.6 & 40.2 & 34.8 & 36.8 & 35.5 & 35.4 & 37.8 & 33.3 & 34.8 & 38.1 & 37.4 & 37.9 & 37.1 \\
\hline
\end{tabular}

\begin{tabular}{|c|c|c|c|c|c|c|c|c|c|c|c|c|c|c|c|}
\hline \multirow[b]{2}{*}{38} & \multicolumn{3}{|c|}{410} & \multicolumn{7}{|c|}{$410 \mathrm{~A}$} & \multicolumn{5}{|c|}{411} \\
\hline & 39 & 39 & 41 & 2 & 2 & 3 & 4 & 4 & 5 & 5 & 6 & 1 & 1 & 2 & 3 \\
\hline 1 & 3 & 6 & 2 & 1 & 4 & 3 & 2 & 4 & 3 & 4 & 2 & 1 & 1 & 1 & 1 \\
\hline $86-91$ & $119-124$ & $28-33$ & $54-59$ & $39-44$ & $25-30$ & $25-30$ & $75 \cdot 80$ & $55-60$ & $85-90$ & $25-30$ & $115-120$ & $7-10$ & $70-75$ & $60-65$ & $37-41$ \\
\hline 48.29 & 49.57 & 51.33 & 51.36 & 49.86 & 49.19 & 47.10 & 47.88 & 46.53 & 47.48 & 47.90 & 45.94 & 47.63 & 48.15 & 47.85 & 47.86 \\
\hline 1.36 & 1.11 & 1.02 & 1.02 & 1.40 & 1.36 & 1.40 & 1.44 & 1.40 & 1.44 & 1.36 & 1.44 & 0.85 & 0.84 & 0.85 & 0.85 \\
\hline 17.27 & 17.31 & 18.01 & 16.60 & 16.00 & 15.91 & 15.77 & 15.86 & 15,23 & 16.40 & 16.26 & 15.04 & 15.11 & 14.71 & 14.63 & 15.43 \\
\hline 3.26 & 5.97 & 4.52 & 3.12 & 3.93 & 3.87 & 4.27 & 4.57 & 4.23 & 3.77 & 4.08 & 5.37 & 3.40 & 2.43 & 2.98 & 2.15 \\
\hline 5.01 & 3.06 & 2.34 & 3.58 & 5.52 & 4.94 & 4.11 & 4.30 & 4.28 & 4.71 & 4.45 & 4.74 & 6.65 & 7.82 & 7.63 & 7.48 \\
\hline 0.17 & 0.16 & 0.11 & 0.16 & 0.17 & 0.16 & 0.11 & 0.11 & 0.11 & 0.17 & 0.17 & 0.15 & 0.17 & 0.15 & 0.17 & 0.15 \\
\hline 7.22 & 3.97 & 3.36 & 5.47 & 7.05 & 7.50 & 8.59 & 7.88 & 7.77 & 7.74 & 8.03 & 7.52 & 10.49 & 11.70 & 11.61 & 11.38 \\
\hline 11.69 & 10.73 & 10.37 & 10.10 & 11.09 & 11.33 & 11.33 & 10.95 & 12.39 & 11.56 & 11.71 & 12.92 & 12.39 & 12.10 & 12.11 & 12.04 \\
\hline 2.97 & 3.93 & 4.19 & 3.93 & 2.88 & 2.70 & 2.90 & 3.00 & 3.00 & 3.00 & 2.90 & 3.00 & 1.98 & 1.98 & 1.98 & 1.98 \\
\hline 0.81 & 1.73 & 2.41 & 1.99 & 0.81 & 0.68 & 0.54 & 0.51 & 0.49 & 0.65 & 0.78 & 0.86 & 0.27 & 0.17 & 0.17 & 0.20 \\
\hline 0.58 & 0.97 & 0.91 & 1.16 & 0.62 & 1.01 & 0.93 & 1.29 & 1.63 & 1.05 & 0.74 & 1.16 & 0.54 & 0.35 & 0.25 & 0.63 \\
\hline 0.88 & 0.85 & 0.90 & 0.84 & 0.90 & 1.22 & 2.25 & 2.28 & 2.21 & 1.74 & 1.43 & 0.74 & 0.46 & 0.10 & 0.16 & 0.24 \\
\hline 0.34 & 0.42 & 0.29 & 0.26 & 0.20 & 0.20 & 0.25 & 0.23 & 0.27 & 0.26 & 0.24 & 0.22 & 0.09 & 0.09 & 0.11 & 0.11 \\
\hline 0.15 & $\therefore$ & - & - & 0.10 & 0.15 & $\therefore$ & 0.05 & $\cdot$ & $\cdot$ & 0.30 & 0.82 & $\cdot$ & $\cdot$ & $\cdot$ & - \\
\hline 100.40 & 99.78 & 99.96 & 99.59 & 100.53 & 100.22 & 99.55 & 100.35 & 99.54 & 99.97 & 100.24 & 99.92 & 100.03 & 100.59 & 100.50 & 100.41 \\
\hline 10.6 & 12.1 & 13.5 & 12.5 & 9.36 & 9.71 & 9.21 & 8.96 & 8.71 & 8.75 & 9.85 & 8.33 & 15.4 & 15.1 & 14.9 & 15.9 \\
\hline 1940 & 1690 & 1610 & 1680 & 1830 & 1900 & 1930 & 1870 & 1990 & 1930 & 1970 & 2050 & 2130 & 2170 & 2150 & 2140 \\
\hline 1730 & 1260 & 1220 & 1390 & 1840 & 1900 & 1750 & 1740 & 1700 & 1710 & 1770 & 1510 & 2160 & 2180 & 2170 & 2180 \\
\hline 19.9 & 31.5 & 40.2 & 33.3 & 18.5 & 17.5 & 17.1 & 17.7 & 18.00 & 18.7 & 18.5 & 18.4 & 10.0 & 9.0 & 8.9 & 9.5 \\
\hline 41.8 & 46.6 & 39.1 & 35.9 & 45.8 & 43.6 & 39.9 & 42.5 & 41.7 & 41.5 & 40.8 & 45.6 & 43.8 & 41.8 & 42.7 & 41.0 \\
\hline 38.3 & 21.9 & 20.7 & 30.8 & 35.7 & 38.9 & 43.0 & 39.8 & 40.3 & 39.8 & 40.7 & 36.0 & 46.2 & 49.2 & 48.4 & 49.5 \\
\hline
\end{tabular}

\begin{tabular}{|c|c|c|c|c|c|c|c|c|c|c|}
\hline \multirow[b]{2}{*}{13} & \multicolumn{4}{|c|}{$412 \mathrm{~A}$} & \multicolumn{4}{|c|}{413} & \multirow[b]{2}{*}{4} & \multirow[b]{2}{*}{5} \\
\hline & 13 & 14 & 14 & 1 & 1 & 2 & 2 & 3 & & \\
\hline 1 & 2 & 2 & 5 & 2 & 2 & 1 & 1 & 1 & 1 & 1 \\
\hline $97-100$ & $30-35$ & $107-112$ & $3-7$ & $75-80$ & $113-118$ & $25-28$ & $72-75$ & $70-75$ & $56-60$ & $55-60$ \\
\hline 50.31 & 50.34 & 50.31 & 49.95 & 64.96 & 47.99 & 47.20 & 47.20 & 48.75 & 46.97 & 47.74 \\
\hline 1.10 & 1.13 & 1.02 & 0.93 & 1.40 & 1.40 & 1.44 & 1.44 & 1.40 & 0.72 & 0.93 \\
\hline 15.06 & 15.38 & 15.60 & 15.72 & 14.75 & 14.15 & 14.77 & 15.16 & 15.70 & 16.59 & 16.16 \\
\hline 1.78 & 1.33 & 1.37 & 1.50 & 2.66 & 3.45 & 1.99 & 2.02 & 0.18 & 3.85 & 4.29 \\
\hline 8.29 & 8.69 & 8.31 & 7.14 & 6.56 & 5.54 & 6.96 & 7.43 & 8.01 & 6.47 & 5.87 \\
\hline 0.18 & 0.18 & 0.17 & 0.15 & 0.16 & 0.11 & 0.14 & 0.16 & 0.16 & 0.16 & 0.18 \\
\hline 7.83 & 7.83 & 8.24 & 8.48 & 10.85 & 10.87 & 11.06 & 10.73 & 10.15 & 10.27 & 9.09 \\
\hline 12.25 & 12.25 & 11.96 & 13.37 & 12.38 & 11.64 & 12.07 & 11.74 & 11.78 & 10.99 & 11.57 \\
\hline 2.40 & 2.25 & 2.33 & 2.18 & 2.31 & 2.25 & 2.33 & 2.33 & 2.33 & 2.40 & 2.48 \\
\hline 0.22 & 0.28 & 0.26 & 0.20 & 0.41 & 0.48 & 0.44 & 0.57 & 0.68 & 0.22 & 0.28 \\
\hline 0.28 & 0.17 & 0.28 & 0.20 & 0.19 & 0.82 & 0.44 & 0.69 & 0.58 & 0.29 & 0.60 \\
\hline 0.36 & 0.26 & 0.22 & 0.40 & 0.70 & 1.48 & 0.91 & 0.73 & 0.14 & 1.19 & 1.09 \\
\hline 0.10 & 0.10 & 0.07 & 0.07 & 0.23 & 0.20 & 0.18 & 0.19 & 0.23 & 0.04 & 0.08 \\
\hline- & - & - & - & - & - & - & - & - & $\cdot$ & - \\
\hline 100.16 & 100.19 & 100.14 & 100.34 & 99.56 & 100.38 & 100.02 & 100.39 & 100.18 & 100.13 & 100.36 \\
\hline 11.51 & 11.62 & 13.1 & 16.3 & 8.86 & 8.5 & 8.64 & 8.91 & 9.55 & 19.7 & 14.7 \\
\hline 1990 & 1996 & 1983 & 2150 & 2140 & 2054 & 2118 & 2080 & 2062 & 1944 & 2000 \\
\hline 2150 & 2238 & 2206 & 2270 & 1940 & 2060 & 1966 & 1930 & 2038 & 1930 & 1990 \\
\hline 12.9 & 12.5 & 12.7 & 12.3 & 12.1 & 12.3 & 12.3 & 12.7 & 14.1 & 11.5 & 12.8 \\
\hline 48.6 & 48.8 & 46.8 & 43.8 & 39.8 & 38.9 & 38.7 & 40.4 & 38.3 & 43.5 & 45.0 \\
\hline 38.5 & 38.7 & 40.5 & 43.9 & 48.1 & 48.8 & 49.0 & 46.9 & 47.6 & 45.0 & 42.2 \\
\hline
\end{tabular}




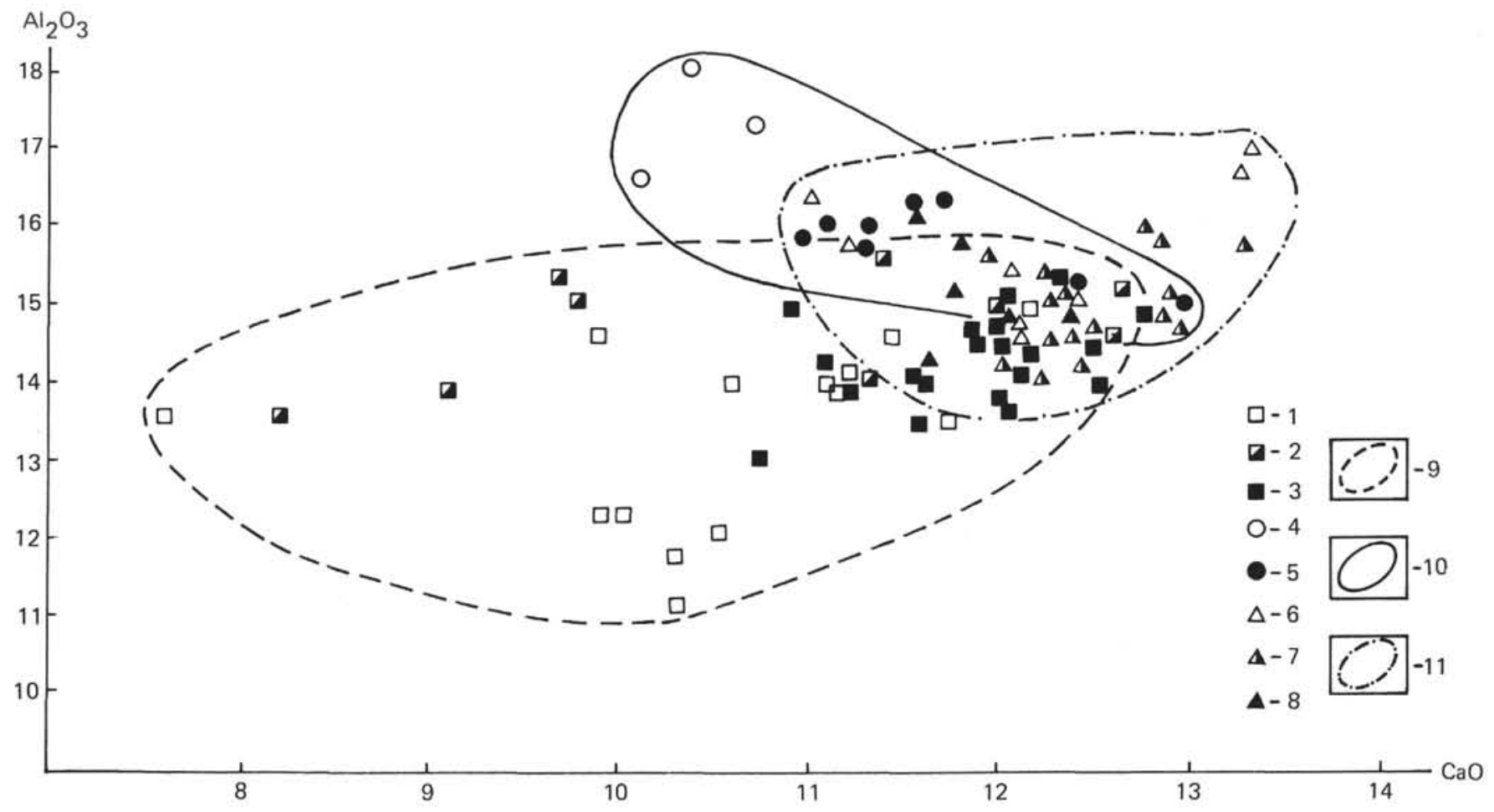

Figure 6. Variation diagram $\mathrm{Al}_{2} \mathrm{O}_{3}-\mathrm{CaO}$. Symbols as in Figure 4.

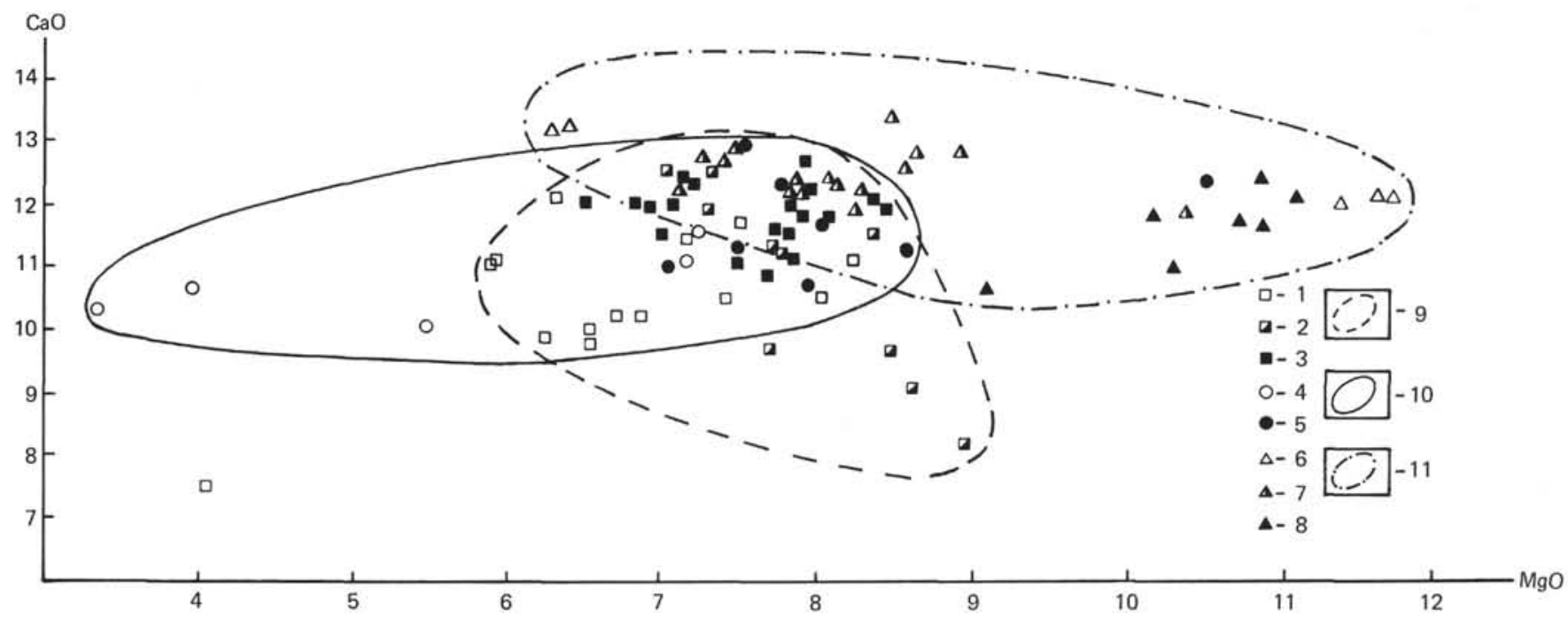

Figure 7. Variation diagram $\mathrm{CaO}-\mathrm{MgO}$. Symbols as in Figure 4.

have erupted in various parts of the Mid-Atlantic Ridge. Judged by most of the elements $\left(\mathrm{TiO}_{2}, \mathrm{Al}_{2} \mathrm{O}_{3}, \mathrm{MgO}, \mathrm{CaO}\right)$, maximum differences were established between basalts of the Mid-Atlantic Ridge and those of the Reykjanes Ridge. Rocks from Hole 407 and some from Hole 408 have an uncommon composition. Basalts from Hole 410 differ from rocks of other holes by their extremely high $\mathrm{K}_{2} \mathrm{O}$ contents.

Table 3 gives average chemical composition of basalts formed in various regions of the Mid-Atlantic Ridge. The table shows that basalts of the Reykjanes Ridge differ significantly from rocks distributed in other regions of the Mid-Atlantic Ridge. The oldest basalts of the Reykjanes Ridge (Hole 407) are similar in composition to the effusives of Iceland, whereas more recent basalts (Hole 409) are analogous to common abyssal tholeiites. All Leg 49 basalts studied are characterized by an anomalously low $\mathrm{Na}{ }_{2} \mathrm{O} / \mathrm{K}_{2} \mathrm{O}$ ratio, as compared with abyssal tholeiites (in which the ratio value reaches 12 to 17 ). Noteworthy is that a portion of the basalts from Hole 407 is characterized by high concentrations of $\mathrm{TiO}_{2}$, as in alkaline basalts of arched uplifts of the oceanic areas (Zolotarev, in press). However, basalts with high titanium contents from the Reykjanes Ridge contain low concentrations of alkaline elements, $\mathrm{K}_{2} \mathrm{O}$ in particular. This makes them similar to the median composition of Icelandic basalts. Considering all other elements, except for $\mathrm{Na}_{2} \mathrm{O}$ and $\mathrm{K}_{2} \mathrm{O}$, basalts with high titanium contents of the Reykjanes 


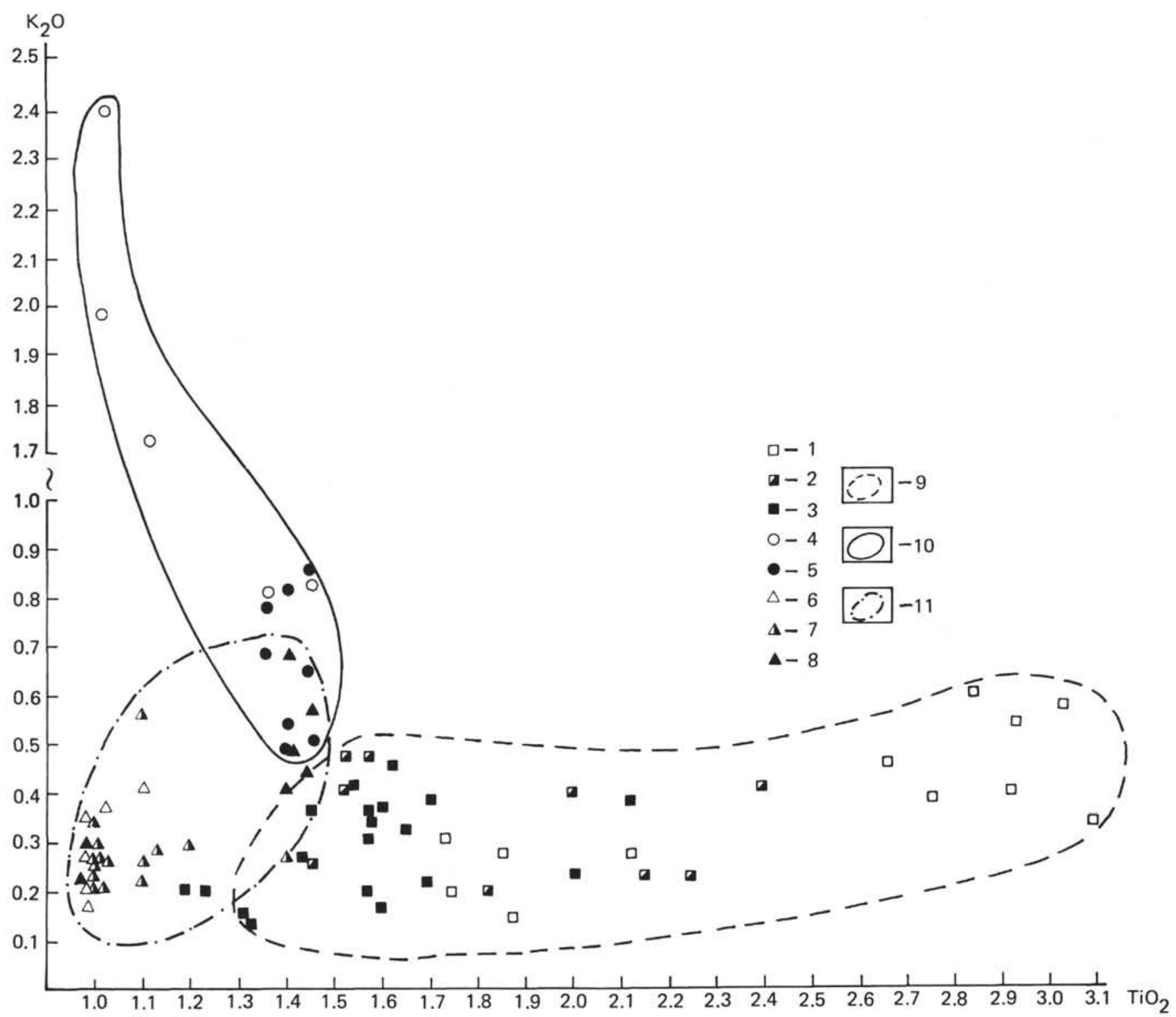

Figure 8. Variation diagram $\mathrm{K}_{2} \mathrm{O}-\mathrm{TiO}_{2}$. Symbols as in Figure 4.

Ridge are surprisingly similar to lunar plagioclase basalts (Woods, 1975). Lower concentrations of alkaline elements in lunar plagioclase basalts may result from the specific character of lunar magmatic eruptions. Under vacuum, readily mobile alkaline elements disappear from the melt in the eruption process.

The basalt study has shown that in the northern part of the Atlantic Ocean (Reykjanes Ridge) there is an agegeochemical specialization of the effusive rocks. The formation of basalts at Site 407 appears to be related to melting of the primary liquid from the undifferentiated parts of the upper mantle. They are characterized by higher concentrations of titanium and iron, and are similar in composition to the undifferentiated lunar mantle. At later stages of magmatic activity (the remaining holes of Leg 49), the melting of basalts proceeded from a differentiated mantle with low levels of titanium and iron.

Another explanation for the established geochemical specialization of the basalts studied should consider the diffe- rent depth of generation of primary melts. Varying degrees of differentiation of the mantle material constituting the asthenospheric levels play a determinative role.

\section{Distribution of Organic Gases in Basalts}

Eighty extracts of natural gases were analyzed and identified as a mixture of nitrogen, hydrogen, and hydrocarbons up to $\mathrm{C}_{5}$ (Table 4). According to the adopted classification, they can be attributed to the group of nitric gases. However, this conclusion does not seem to be well founded because the chosen recovery methodology does not exclude sample contamination with nitrogen in the air. Contamination seems most probable, because all the extracts of gases contained various amounts of free oxygen. If one excludes surface atmosphere gases $\left(\mathrm{O}_{2}\right.$ and $\left.\mathrm{N}_{2}\right)$ from consideration, the gases contained in the sample basalts are hydrogenous, based on their chemical composition. The $\mathrm{CO}_{2}$ content in the extracts under study are, as a rule, below sensitivity of the catarome- 
TABLE 3

Average Chemical Composition of Basalts From the Mid-Atlantic Ridge, Iceland, and Effusive Lunar Rocks (wt. \%)

\begin{tabular}{|c|c|c|c|c|c|c|}
\hline Components & 1 & 2 & 3 & 4 & 5 & 6 \\
\hline $\mathrm{SiO}_{2}$ & 47.71 & 48.67 & 49.05 & 47.72 & 47.10 & 47.33 \\
\hline $\mathrm{TiO}_{2}$ & 1.93 & 1.32 & 1.06 & 2.96 & 3.17 & 2.35 \\
\hline $\mathrm{Al}_{2} \mathrm{O}_{3}$ & 13.98 & 16.26 & 15.17 & 11.78 & 18.20 & 15.09 \\
\hline $\mathrm{FeO}$ & 12.33 & 8.06 & 9.47 & 16.15 & 17.40 & 11.18 \\
\hline $\mathrm{MnO}$ & 0.18 & 0.14 & 0.18 & 1.28 & 0.24 & 0.20 \\
\hline $\mathrm{MgO}$ & 7.35 & 6.86 & 99.01 & 6.55 & 6.80 & 6.89 \\
\hline $\mathrm{CaO}$ & 11.17 & 11.33 & 12.33 & 10.76 & 11.40 & 11.19 \\
\hline $\mathrm{Na}_{2} \mathrm{O}$ & 2.49 & 3.17 & 2.26 & 2.60 & 0.64 & 2.61 \\
\hline $\mathrm{K}_{2} \mathrm{O}$ & 0.35 & 1.01 & 0.31 & 0.49 & 0.07 & 0.57 \\
\hline $\mathrm{FeO} / \mathrm{MgO}$ & 1.68 & 1.18 & 1.05 & 2.46 & 2.56 & 1.62 \\
\hline $\mathrm{Na}_{2} \mathrm{O} / \mathrm{K}_{2} \mathrm{O}$ & 7.10 & 3.14 & 7.30 & 5.20 & 9.10 & 4.58 \\
\hline
\end{tabular}

Note: 1 - basalts from Holes 407, 408 and 409 (43 analyses); 2 - basalts from Holes 410 and $410 \mathrm{~A}$ (13 analyses); 3 - basalts from Holes 411, 412A and 413 (29 analyses); 4 - titanium-rich basalts from Hole 407 ( 5 analyses); 5 - plagioclase basalt from the Moon ("Apollo-12" J. A. Wood, 1975); 6 - basalts from Iceland (17 analyses, B. P. Zolotarev, in press).

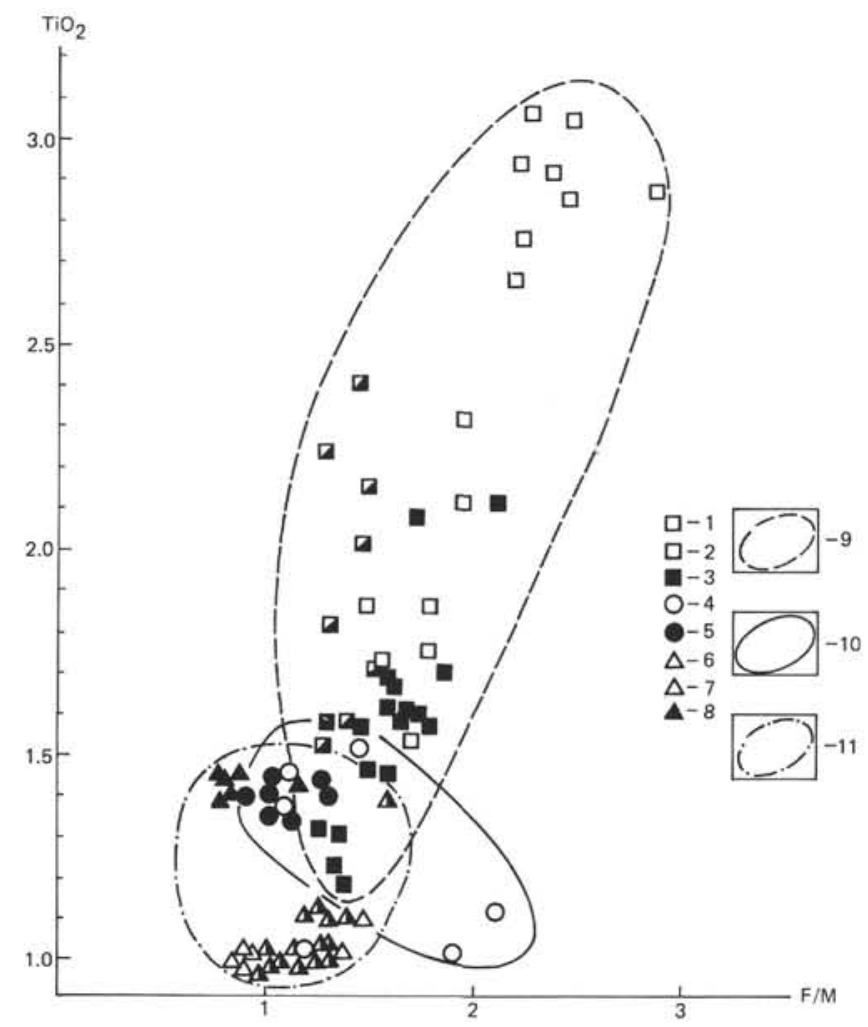

Figure 9. Variation diagram $\mathrm{TiO}_{2}-(F / M) . \mathrm{F}=$ iron total recalculated to $\mathrm{FeO} ; M=M g O$. Other symbols as in Figure 4.

ter $(0.05 \%) . \mathrm{CO}_{2}$ was recognized in the gas of only one sample.

Hydrocarbons, including a set of the components up to $\mathrm{C}_{5}$, were recorded in 5 of the 80 basalt samples. The usual components of the gases studied were $\mathrm{CH}_{4}$ and $\mathrm{C}_{2} \mathrm{H}_{6}$, since they are the most stable hydrocarbons of the alkanes.

The total average gas content in the basalts studied (nitrogen excluded) is not large: tentatively, 1.0 to $1.2 \mathrm{~cm}^{3} / \mathrm{kg}$. They consist of $\mathrm{H}_{2}, 1.0$ to $1.2 \mathrm{~cm}^{3} / \mathrm{kg} ; \mathrm{CH}_{4} 0.025 \mathrm{~cm}^{3} / \mathrm{kg}$; and $\mathrm{C}_{2} \mathrm{H}_{8} 0.012 \mathrm{~cm}^{3} / \mathrm{kg}$. However, the interval of values of these gases is significant, i.e., $\mathrm{H}_{2} 0.1$ to $3.3 \mathrm{~cm}^{3} / \mathrm{kg} ; \mathrm{CH}_{4}$, 0.007 to $0.08 \mathrm{~cm}^{3} / \mathrm{kg} ; \mathrm{C}_{2} \mathrm{H}_{2}, 0.0008$ to $0.008 \mathrm{~cm}^{3} / \mathrm{kg}$; and $\mathrm{C}_{2} \mathrm{H}_{8}, 0.0006$ to $0.008 \mathrm{~cm}^{3} / \mathrm{kg}$. The variability of all individual gas contents is greatest in samples from Holes 407 , 408 , and 409. This range is somewhat narrower in samples from Holes 411, 412A, and 413, and the narrowest in basalts of Holes 410, and 410A. The average contents of all individual gases (except for hydrogen) change respectively in the same way (Table 5).

Histograms of distribution of gas contents compiled according to regional features show no observable changes in the contents or concentrations of gases (Figure 14). By composition and content, these gases are similar to those from basalts of the Kuril island arc of the southwestern sector of the Pacific Ocean (Zolotarev et al., 1976), Iceland (Zolotarev et al., in press b), and the Mid-Atlantic Ridge (Zolotarev et al., in press a).

The chemical composition of gases contained in basalts does not depend on the chemical composition of the primary melt. The gas saturation of basalts appears to depend only on the form of volcanism manifested (effusive, aerial, effusive underwater, or intrusive), and the degree of epimagmatic changes of the rocks.

\section{REFERENCES}

de La Roche, H. and Govindaraju, K., 1973. Rapport (1972) sur quatre standards geóchimiques de l'Association Nationale de la Recherche Technique: diorite DR-N, serpentinite UB/N, bauxite BX-N et disthene DT-N; Extrait du Bulletin de la Societe Francaise de Ceramique, no. 1000, p. 49-75.

de La Roche, H. and Letterier, J., 1973. Transposition du tetraedre mineŕalogique de Yoder et Tilly dans un diagramme chimique de classification de roches basaltiques, C. R. Acad. Sci., Paris, v. 276, p. $135-159$.

Gottini, V., 1970. Serial character of the volcanic rocks of Pantelleria, Bull. Volcanol., v. 33, p. 818-827.

Kuno, H., 1966. Lateral variation of basalt magma across continental margins and island areas, Geol. Surv., Canada, no. 2, p. 324-339.

Miyashiro, A., 1975. Classification characteristic and origin of ophiolites, Journal of Geology, v. 83, p. 249-281.

Roubalt, M., de La Roche, H., and Govindaraju, K., 1970. Etat actuel (1970) des etudes cooperatives eur les standards geóchemiques du Centre de Recherches Petrographiques et Geochimiques, Sciences de la Terre, v. 15, p. 351-393.

Wood, G. A., 1975. Review of the Lunar rock types and comparison of the Lunar and Earth crusts. In "Cosmochemistry of the Moon and the Planets; Moscow ("Nauka").

Yoder, H. S. and Tilly, C. E., 1962. Origin of basalt magma, J. Petrol., v. 3, p. 342-532.

Zolotarev, B. P., in press. Petrochemistry of basalts of the recent ocean, Moscow ("Nauka').

Zolotarev, B. P., Voitov, G. I., and Cherevichnaya, A. F., 1976. On gases of young basaltoids of some islands of the south 


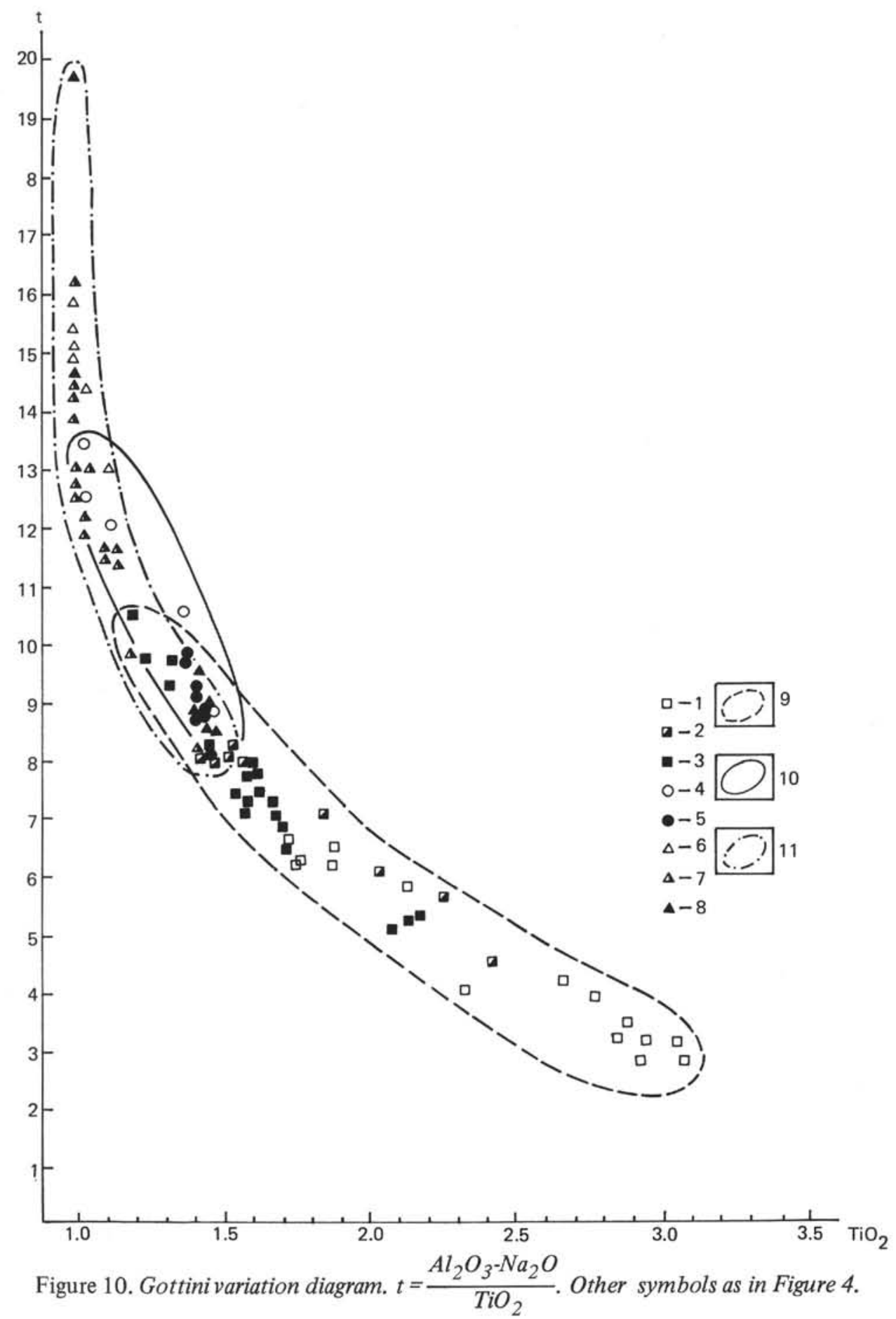

western sector of the Pacific Ocean, Doklady Akad. Nauk SSSR, v. 229 , p. $721-724$.

Zolotarev, B. P., Voitov, G. I., Sarkisyan, I. S., and Cherevichnaya, L. F., in press a. Distribution of gases and bitumens in basalts from Holes 395 and 396, Leg 45. In Melson, W. G., Rabinowitz, P. D., et al., Initial Reports of the Deep Sea Drill- ing Project, v. 45: Washington (U.S. Government Printing Office).

Zolotarev, B. P., Trifonov, V. G., Voitov, G. I., and Cherevichnaya, L. F., in press b. On gases included in volcanic rocks of Iceland, Doklady Akad. Nauk SSSR. 


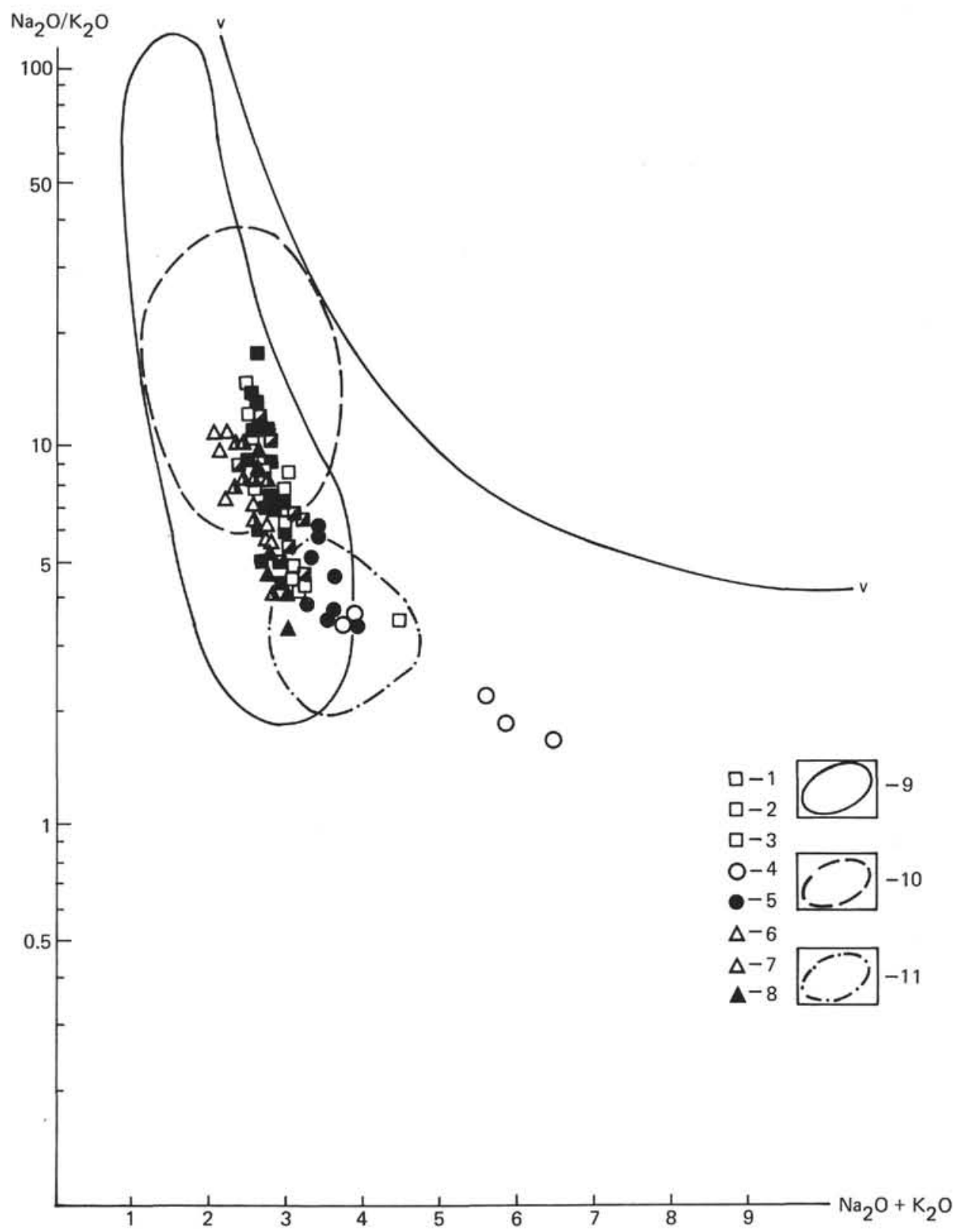

Figure 11. Miyashiro variation diagram. $V-V=$ the boundary line separating fresh rocks from those altered by epimagmatic processes. $9=$ Icelandic tholeiite field, $10=$ abyssal tholeiite field, 11 - field of alkaline rocks of the Atlantic islands; other symbols as in Figure 4. 


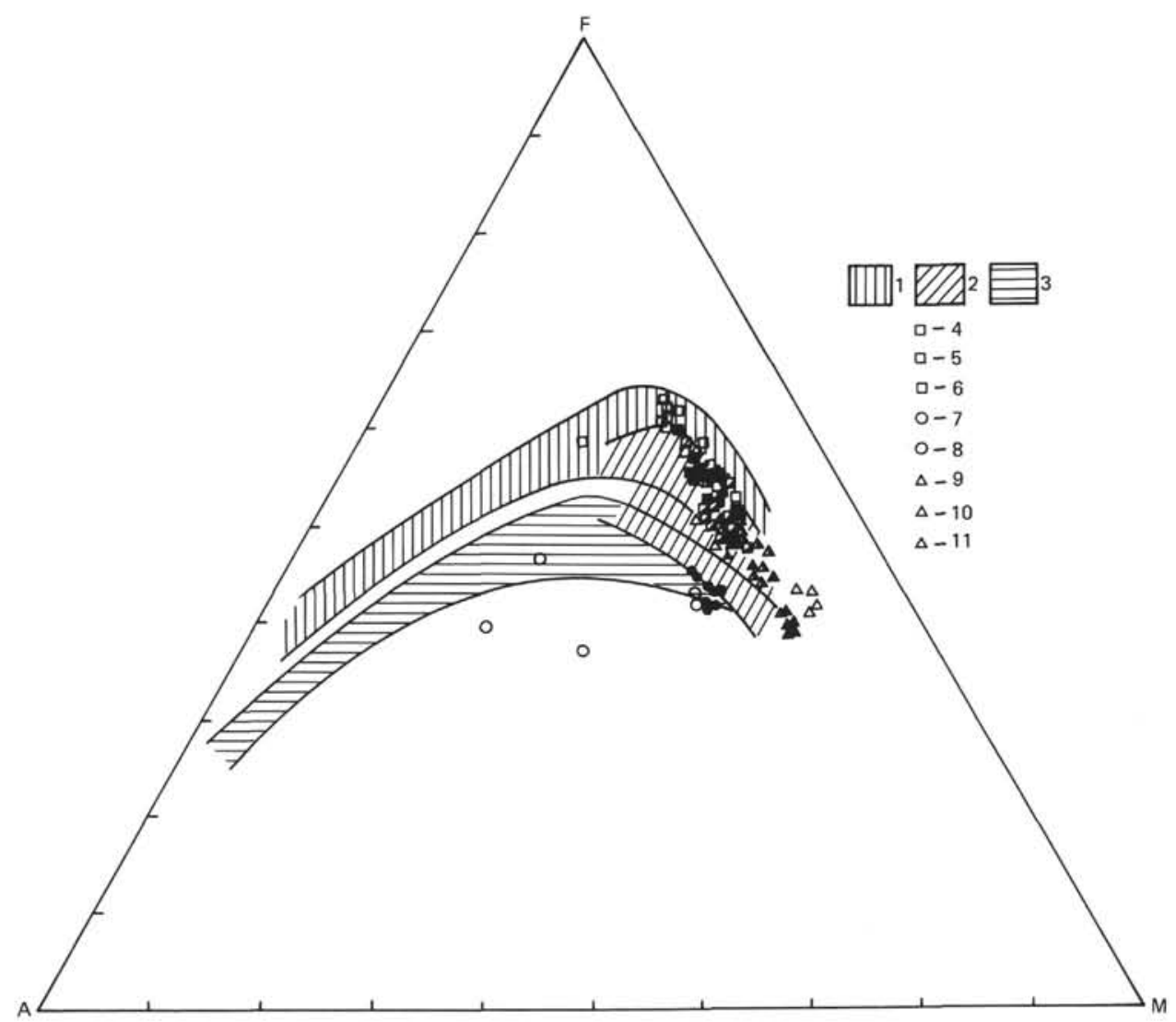

Figure 12. Three-component $A F M$ diagram. $\mathrm{A}=\left(\mathrm{Na} a_{2} \mathrm{O}+\mathrm{K}_{2} \mathrm{O}\right), \mathrm{F}=\left(\mathrm{Fe}_{2} \mathrm{O}_{3}+\mathrm{FeO}\right.$, recalculated to $\mathrm{FeO}), M=(\mathrm{MgO}), 1$ = tholeiite field, 2 = high-alumina field, $3=$ alkaline olivine-basalt field. Other symbols as in Figure 4. 
B. P. ZOLOTAREV, D. YA. CHOPOROV, G. I. VOITOV

TABLE 4

Chemical Composition of Gases in Basalts From Holes 407, 408, 409, 410, 410A, 411, 412A, and 413

\begin{tabular}{|c|c|c|c|c|c|c|c|c|c|c|c|}
\hline \multirow[b]{2}{*}{$\begin{array}{c}\text { Sample } \\
\text { (Interval in } \mathrm{cm} \text { ) }\end{array}$} & \multicolumn{11}{|c|}{ Chemical Composition of Gases; Numerator $\%$, Denominator $\mathrm{cm}^{3} / \mathrm{kg}$} \\
\hline & $\mathrm{H}_{2}$ & $\mathrm{O}_{2}$ & & $\mathrm{CO}_{2}$ & $\mathrm{CH}_{4}$ & $\mathrm{C}_{2} \mathrm{H}_{6}$ & $\mathrm{C}_{2} \mathrm{H}_{4}$ & $\mathrm{C}_{3} \mathrm{H}_{8}$ & $\mathrm{C}_{3} \mathrm{H}_{6}$ & n $\mathrm{C}_{4} \mathrm{H}_{10}$ & $\mathrm{n} \mathrm{C}_{4} \mathrm{H}_{10}$ \\
\hline 2 & 3 & 4 & 5 & 6 & 7 & 8 & 9 & 10 & 11 & 12 & 13 \\
\hline $\begin{array}{l}\text { Hole } 407 \\
40735-1,30-33\end{array}$ & - & - & - & - & $\frac{77.77}{0.03}$ & $\frac{11.11}{0.0043}$ & - & $\frac{11.11}{0.0043}$ & - & - & - \\
\hline $40736-1,20-25$ & $\frac{2.436}{0.937}$ & - & $\frac{97.43}{35.50}$ & - & $\frac{0.114}{0.044}$ & $\frac{0.011}{0.004}$ & - & $\frac{0.006}{0.0016}$ & - & - & - \\
\hline $40736-3,95-97$ & $\frac{98.54}{1.098}$ & - & - & - & $\frac{1.36}{0.015}$ & $\frac{0.102}{0.001}$ & - & - & - & - & - \\
\hline $40737-1,112-117$ & $\frac{2.199}{1.125}$ & - & $\frac{97.77}{46.77}$ & - & $\frac{0.048}{0.011}$ & $\frac{0.006}{0.003}$ & - & $\frac{0.003}{0.0015}$ & - & - & - \\
\hline $40737-2,109-111$ & - & - & $\frac{94.99}{88.89}$ & - & $\frac{5.01}{0.009}$ & - & - & - & - & - & - \\
\hline $40738-2,15-17$ & $\frac{0.148}{0.139}$ & - & $\frac{99.22}{21.29}$ & - & $\frac{0.103}{0.022}$ & $\frac{0.0013}{0.0028}$ & - & $\frac{0.0013}{0.0028}$ & - & - & - \\
\hline $40739-2,100-105$ & $\frac{0.53}{1.438}$ & - & $\frac{99.41}{277.25}$ & - & $\frac{0.009}{0.023}$ & $\frac{0.001}{0.0025}$ & - & - & - & - & - \\
\hline $40740-1,70-75$ & $\frac{3.38}{0.641}$ & - & $\frac{96.44}{14.74}$ & - & $\frac{0.12}{0.025}$ & $\frac{0.014}{0.0039}$ & - & - & - & - & - \\
\hline $407-42-2,35-40$ & $\frac{1.011}{1.212}$ & - & $\frac{98.90}{119.0}$ & - & $\frac{0.0234}{0.028}$ & $\frac{0.0039}{0.0047}$ & $\frac{0.0026}{0.003}$ & $\frac{0.0039}{0.0047}$ & - & $\frac{0.0013}{0.0015}$ & $\frac{0.0039}{0.0047}$ \\
\hline $407-44-1,60-65$ & $\frac{0.689}{0.895}$ & - & $\frac{99.85}{129.0}$ & - & $\frac{0.0173}{0.022}$ & $\frac{0.0017}{0.0021}$ & - & - & - & - & - \\
\hline $407-45-1,55-60$ & $\frac{1.025}{2.16}$ & - & $\frac{98.95}{208.3}$ & - & $\frac{0.0165}{0.0348}$ & $\frac{0.0016}{0.0075}$ & - & - & - & - & - \\
\hline $407-45-2,0-5$ & $\frac{97.90}{0.99}$ & - & & - & $\frac{0.855}{0.0188}$ & $\frac{0.145}{0.00145}$ & - & $\frac{0.144}{0.00144}$ & - & - & - \\
\hline $\begin{array}{l}407-47-3,77-80 \\
\text { Hole } 408\end{array}$ & $\frac{0.576}{0.395}$ & - & $\frac{99.40}{68.50}$ & - & $\frac{0.05}{0.0342}$ & $\frac{0.0057}{0.0039}$ & - & $\frac{0.0025}{0.0017}$ & - & $\frac{0.0036}{0.0026}$ & $\frac{0.0029}{0.002}$ \\
\hline $40836-2,83-85$ & $\frac{2.24}{3.14}$ & - & $\frac{97.60}{137.0}$ & - & $\frac{0.033}{0.0646}$ & $\frac{0.018}{0.0025}$ & - & - & - & - & - \\
\hline $40836-3,7-10$ & $\frac{2.2}{0.785}$ & - & $\frac{97.40}{35.70}$ & - & $\frac{0.129}{0.472}$ & $\frac{0.0312}{0.114}$ & $\frac{0.156}{0.057}$ & $\frac{0.156}{0.057}$ & $\frac{0.0195}{0.0715}$ & $\frac{0.0078}{0.028}$ & $\frac{0.0156}{0.057}$ \\
\hline $40836-5,34-37$ & & - & $\frac{99.99}{264.0}$ & - & $\frac{0.0058}{0.0154}$ & $\frac{0.0108}{0.0029}$ & - & - & - & - & - \\
\hline $40837-1,74-77$ & $\frac{0.481}{2.188}$ & - & $\frac{99.51}{452.08}$ & - & $\frac{0.011}{0.050}$ & $\frac{0.0014}{0.0063}$ & - & - & - & - & - \\
\hline $40837-2,89-91$ & $\frac{0.967}{2.232}$ & - & $\frac{99.01}{228.57}$ & - & $\frac{0.020}{0.0464}$ & $\frac{0.0015}{0.0036}$ & - & - & - & - & - \\
\hline $40837-3,33-35$ & $\frac{5.92}{2.417}$ & - & $\frac{93.96}{38.33}$ & - & $\frac{0.098}{0.040}$ & $\frac{0.0123}{0.005}$ & - & $\frac{0.0053}{0.0022}$ & - & - & - \\
\hline $40838-1,66-68$ & $\frac{1.695}{0.375}$ & - & $\frac{97.95}{21.67}$ & - & $\frac{0.354}{0.078}$ & $\frac{0.0049}{0.0011}$ & - & - & - & - & - \\
\hline $40838-2,38-40$ & $\frac{0.988}{0.298}$ & - & $\frac{98.845}{29.762}$ & - & $\frac{0.1304}{0.0393}$ & $\frac{0.0158}{0.0048}$ & $\frac{0.0079}{0.0024}$ & $\frac{0.0118}{0.0086}$ & - & - & - \\
\hline $\begin{array}{l}40838-3,95-97 \\
\text { Hole } 409\end{array}$ & $\frac{3.740}{1.544}$ & - & $\frac{96.174}{39.706}$ & - & $\frac{0.0784}{0.0324}$ & $\frac{0.0071}{0.0029}$ & - & - & - & - & - \\
\hline $\begin{array}{l}\text { Hole 409 } \\
4097-6,135-140\end{array}$ & $\frac{3.996}{1.157}$ & - & $\frac{95.913}{27.78}$ & - & $\frac{0.0799}{0.023}$ & $\frac{0.0064}{0.0019}$ & - & $\frac{0.0042}{0.0012}$ & - & - & - \\
\hline $4099-2,95-100$ & $\frac{0.369}{0.658}$ & - & $\frac{99.61}{177.63}$ & - & $\frac{0.0192}{0.0342}$ & $\frac{0.0015}{0.0026}$ & - & - & - & - & - \\
\hline $409-10-1-50-55$ & $\frac{10.592}{2.375}$ & - & $\frac{89.196}{20.00}$ & - & $\frac{0.1895}{0.0425}$ & $\frac{0.0167}{0.0038}$ & - & $\frac{0.0056}{0.0013}$ & - & - & - \\
\hline $40910-4,95-100$ & $\frac{1.419}{2.813}$ & - & $\frac{98.556}{195.31}$ & - & $\frac{0.0228}{0.0453}$ & $\frac{0.0016}{0.0031}$ & - & - & - & - & - \\
\hline $40911-3,70-75$ & $\frac{1.848}{0.833}$ & - & $\frac{98.102}{44.23}$ & - & $\frac{0.0426}{0.0192}$ & $\frac{0.0057}{0.0026}$ & - & $\frac{0.0018}{0.0008}$ & - & - & - \\
\hline
\end{tabular}


TABLE 4 - Continued

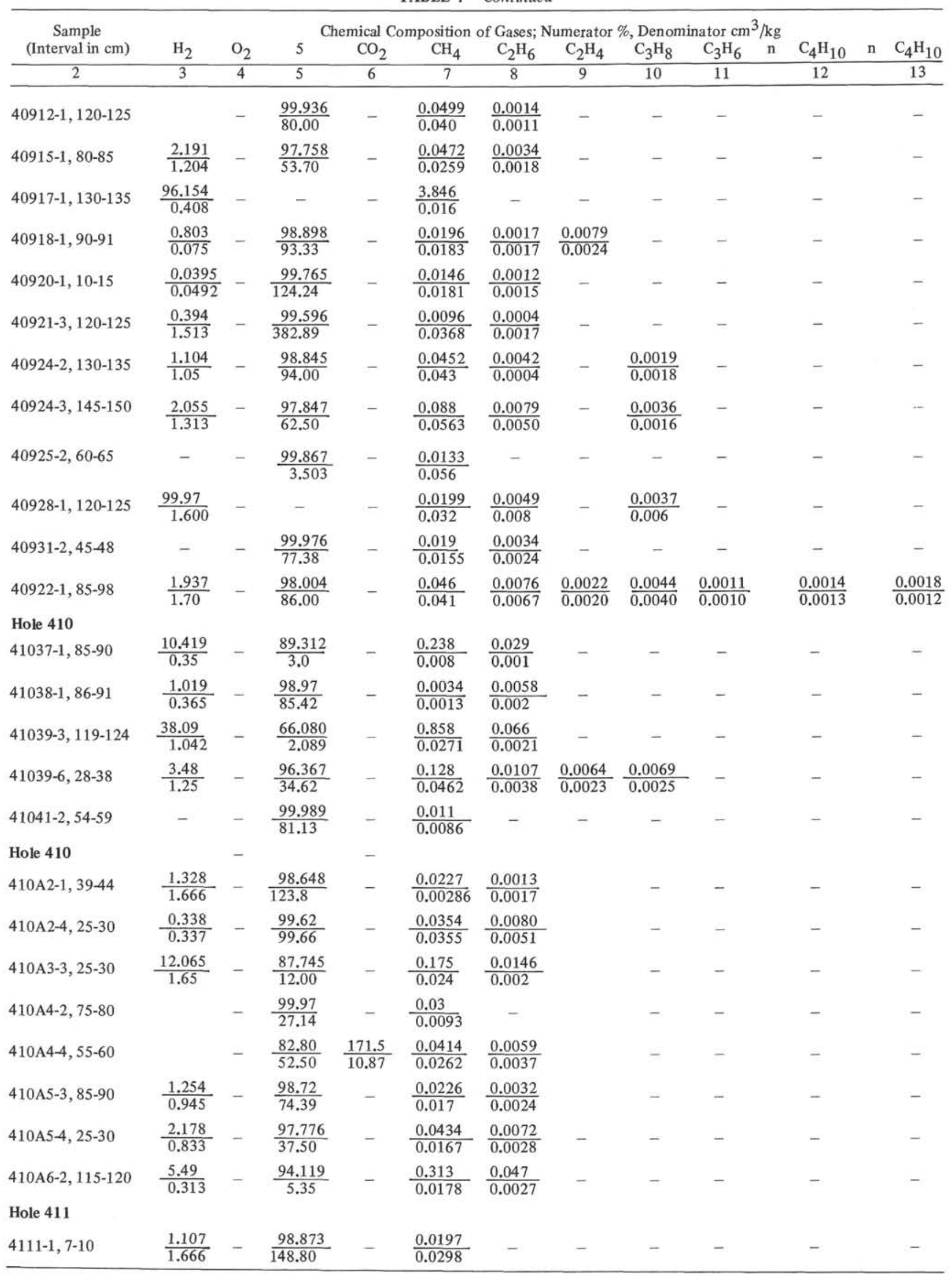


TABLE 4 - Continued

\begin{tabular}{|c|c|c|c|c|c|c|c|c|c|c|c|}
\hline \multirow{2}{*}{$\begin{array}{c}\text { Sample } \\
\text { (Interval in } \mathrm{cm} \text { ) }\end{array}$} & \multicolumn{11}{|c|}{ Chemical Composition of Gases; Numerator $\%$, Denominator $\mathrm{cm}^{3} / \mathrm{kg}$} \\
\hline & $\mathrm{H}_{2}$ & $\mathrm{O}_{2}$ & & $\mathrm{CO}_{2}$ & $\mathrm{CH}_{4}$ & $\mathrm{C}_{2} \mathrm{H}_{6}$ & $\mathrm{C}_{2} \mathrm{H}_{4}$ & $\mathrm{C}_{3} \mathrm{H}_{8}$ & $\mathrm{C}_{3} \mathrm{H}_{6}$ & $\mathrm{n} \mathrm{C}_{4} \mathrm{H}_{10}$ & n $\quad \mathrm{C}_{4} \mathrm{H}_{10}$ \\
\hline 2 & 3 & 4 & 5 & 6 & 7 & 8 & 9 & 10 & 11 & 12 & 13 \\
\hline $4111-1,70-75$ & $\frac{2.065}{1.250}$ & - & $\frac{97.907}{59.25}$ & - & $\frac{0.0260}{0.0157}$ & $\frac{0.0021}{0.0013}$ & - & - & - & - & - \\
\hline $4112-1,60-65$ & $\frac{0.887}{0.151}$ & - & $\frac{99.099}{168.75}$ & - & $\frac{0.0135}{0.0229}$ & $\frac{0.009}{0.0015}$ & - & - & - & - & - \\
\hline $4113-1,37-41$ & $\frac{3.053}{0.58}$ & - & $\frac{96.866}{46.00}$ & - & $\frac{0.0505}{0.024}$ & $\frac{0.0029}{0.0014}$ & - & - & - & - & - \\
\hline $4113-1,143-145$ & $\frac{0.38}{0.859}$ & - & $\frac{99.605}{225.0}$ & - & $\frac{0.0131}{0.0297}$ & $\frac{0.0009}{0.0022}$ & - & - & - & - & - \\
\hline $4114-1,84-88$ & $\frac{1.73}{0.807}$ & - & $\frac{98.22}{45.83}$ & - & $\frac{0.037}{0.0171}$ & $\frac{0.0044}{0.0021}$ & $\frac{0.0013}{0.006}$ & $\frac{0.0028}{0.0013}$ & $\frac{0.0015}{0.0007}$ & - & - \\
\hline \multicolumn{12}{|l|}{ Hole $412 \mathrm{~A}$} \\
\hline $412 \mathrm{~A} 1-1,16-20$ & $\frac{91.32}{0.208}$ & - & - & - & $\frac{7.305}{0.0017}$ & $\frac{1.369}{0.0031}$ & - & - & - & - & - \\
\hline $412 \mathrm{~A} 2-1,71-75$ & $\frac{0.858}{1.285}$ & - & $\frac{99,133}{149.30}$ & - & $\frac{0.0115}{0.024}$ & $\frac{0.0013}{0.0021}$ & - & - & - & - & - \\
\hline $412 \mathrm{~A} 3-1,47-50$ & $\frac{2.952}{1.521}$ & - & $\frac{96.983}{50.00}$ & - & $\frac{0.0611}{0.0315}$ & $\frac{0.0042}{0.0022}$ & - & - & - & - & - \\
\hline $412 \mathrm{~A} 3-2,45-50$ & $\frac{97.50}{1.547}$ & - & - & - & $\frac{2.175}{0.0345}$ & $\frac{0.225}{0.0086}$ & $\frac{0.0775}{0.0015}$ & $\frac{0.0775}{0.0015}$ & - & - & - \\
\hline $412 \mathrm{~A} 3-3,42-45$ & $\frac{0.949}{0.921}$ & - & $\frac{99.031}{96.05}$ & - & $\frac{0.0189}{0.0184}$ & $\frac{0.0027}{0.0026}$ & - & - & - & - & - \\
\hline $412 \mathrm{~A}-51,35-40$ & $\frac{0.96}{0.955}$ & - & $\frac{99.01}{98.52}$ & - & $\frac{0.0026}{0.0257}$ & $\frac{0.0022}{0.0022}$ & - & $\frac{0.0002}{0.0024}$ & - & - & - \\
\hline $412 \mathrm{~A} 6-1,85-90$ & $\frac{2.001}{0.363}$ & - & $\frac{97.862}{17.74}$ & - & $\frac{0.116}{0.0209}$ & $\frac{0.0151}{0.0027}$ & - & $\frac{0.0058}{0.00102}$ & - & - & - \\
\hline $412 \mathrm{~A} 7-2,130-135$ & $\frac{1.797}{1.16}$ & - & $\frac{98.166}{63.39}$ & - & $\frac{0.0345}{0.0228}$ & $\frac{0.0017}{0.0012}$ & - & - & - & - & - \\
\hline 412 A8-1, $90-95$ & $\frac{98.159}{1.176}$ & - & - & - & $\frac{1.718}{0.0205}$ & $\frac{0.122}{0.0015}$ & - & - & - & - & - \\
\hline $412 \mathrm{~A} 9-1,130-135$ & $\frac{11.847}{1.688}$ & - & $\frac{87.758}{12.50}$ & - & $\frac{0.307}{0.0438}$ & $\frac{0.0351}{0.005}$ & - & $\frac{0.263}{0.0037}$ & $\frac{0.0087}{0.00125}$ & $\frac{0.0174}{0.0025}$ & - \\
\hline $412 \mathrm{~A} 11-2,140-145$ & $\frac{1.706}{0.801}$ & - & $\frac{98.264}{46.15}$ & - & $\frac{0.030}{0.0141}$ & - & - & - & - & - & - \\
\hline $412 \mathrm{~A} 11-3,5$ & $\frac{6.426}{1.031}$ & - & $\frac{93.469}{15.00}$ & - & $\frac{0.0973}{0.0156}$ & $\frac{0.0077}{0.0013}$ & - & - & - & - & - \\
\hline $412 \mathrm{~A} 13-1,9-7100$ & $\frac{2.095}{0.662}$ & - & $\frac{97.79}{29.66}$ & - & $\frac{0.093}{0.0294}$ & $\frac{0.0093}{0.0029}$ & $\frac{0.0046}{0.0015}$ & $\frac{0.0093}{0.0029}$ & - & - & - \\
\hline $412 \mathrm{~A} 13-2,30-35$ & $\frac{5.53}{2.656}$ & - & $\frac{94.36}{45.31}$ & - & $\frac{0.976}{0.0469}$ & $\frac{0.0098}{0.0047}$ & - & - & - & - & - \\
\hline $412 \mathrm{~A} 14-2,107-112$ & & - & $\frac{99.996}{444.19}$ & - & $\frac{0.004}{0.016}$ & - & - & - & - & - & - \\
\hline $412 \mathrm{~A}-145,3-7$ & $\frac{0.748}{0.724}$ & - & $\frac{99.217}{101.71}$ & - & $\frac{0.0299}{0.0289}$ & $\frac{0.0040}{0.0039}$ & - & $\frac{0.0013}{0.0013}$ & - & - & - \\
\hline \multicolumn{12}{|l|}{ Hole 413} \\
\hline $4131-2,75-80$ & $\frac{4.106}{1.50}$ & - & $\frac{95.809}{35.00}$ & - & $\frac{0.0718}{0.0263}$ & $\frac{0.0047}{0.0018}$ & $\frac{0.0034}{0.0013}$ & $\frac{0.0047}{0.0016}$ & - & - & - \\
\hline $4131-2,113-118$ & $\frac{0.826}{0.156}$ & - & $\frac{99.119}{18.75}$ & - & $\frac{0.055}{0.0104}$ & - & - & - & - & - & - \\
\hline $4132-1,25-28$ & $\frac{0.144}{0.135}$ & - & $\frac{99.816}{93.75}$ & - & $\frac{0.0354}{0.033}$ & $\frac{0.0044}{0.0042}$ & - & - & - & - & - \\
\hline $4122-1,72-75$ & $\frac{1.738}{1.513}$ & - & $\frac{98.218}{85.52}$ & - & $\frac{0.0423}{0.0368}$ & $\frac{0.0021}{0.0018}$ & - & - & - & - & - \\
\hline $4133-1,70-75$ & $\frac{4.756}{0.641}$ & - & $\frac{95.129}{12.82}$ & - & $\frac{0.105}{0.0141}$ & $\frac{0.0095}{0.0013}$ & - & - & - & - & - \\
\hline $4134-1,56-60$ & $\frac{1.768}{0.804}$ & - & $\frac{98.197}{44.64}$ & - & $\frac{0.035}{0.0161}$ & - & - & - & - & - & - \\
\hline
\end{tabular}




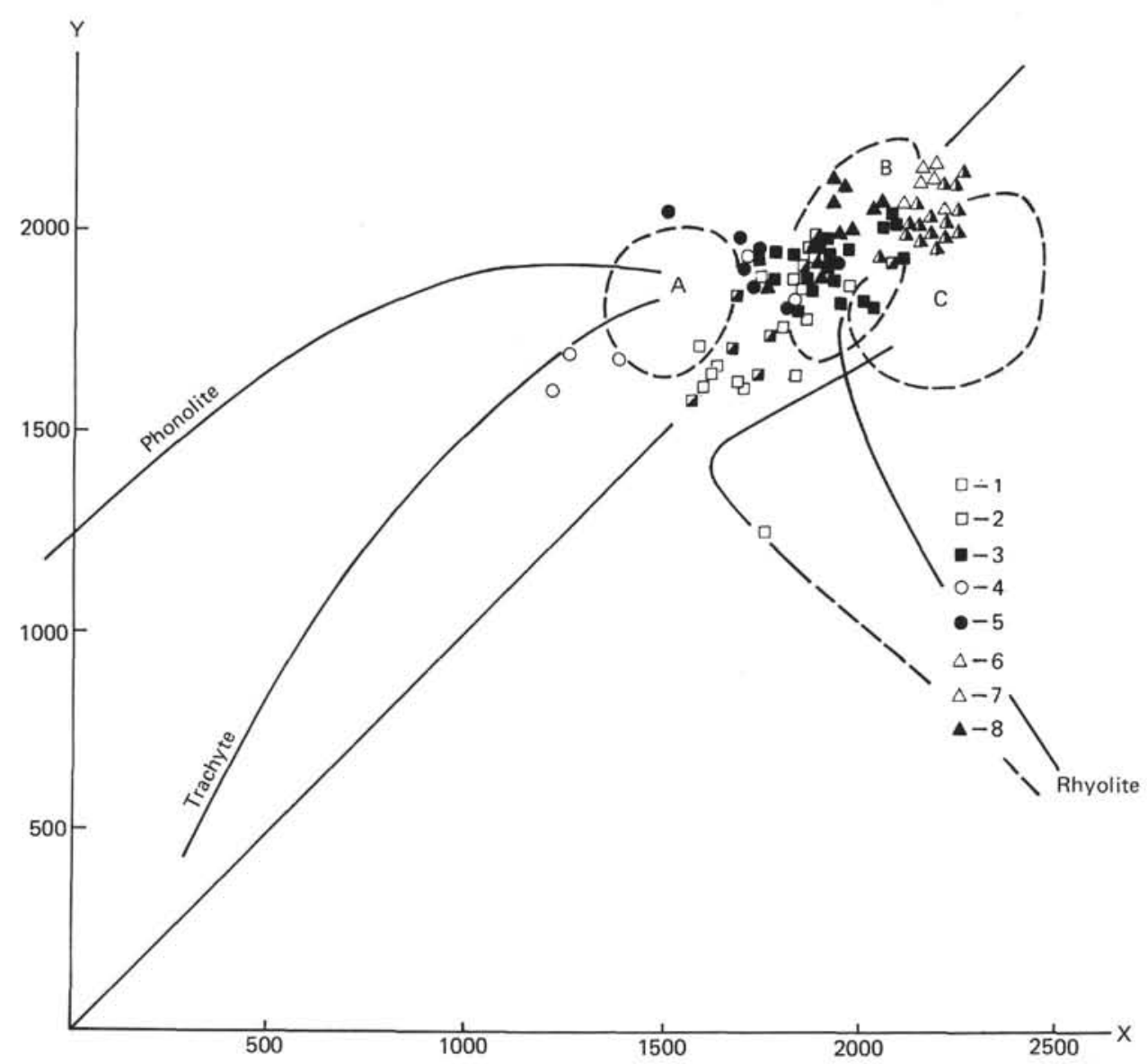

Figure 13. H. de La Roche and J. Letterier variation diagram. $X=4 \mathrm{Si}-I I(\mathrm{Na}+\mathrm{K})-2(\mathrm{Fe}+$ Ti), $y=6 C a+2 M g+A 1$. Elements are presented in atomic proportions. $A=$ alkaline olivine-basalt field, $B=$ high-alumina field, $C=$ tholeiite field. Other symbols as in Figure 4.

TABLE 5

Average Chemical Composition of Gases in Basalts From Holes 407, 408, 409, $410410 \mathrm{~A}, 411,412 \mathrm{~A}$, and 413

\begin{tabular}{|c|c|c|c|c|c|c|c|c|c|c|c|}
\hline \multirow[b]{2}{*}{ Holes } & \multirow{2}{*}{$\begin{array}{l}\text { Number } \\
\text { of } \\
\text { Samples }\end{array}$} & \multicolumn{10}{|c|}{ Chemical Composition of Gases, Numerator $\%$, Denominator $\mathrm{cm}^{3} / \mathrm{kg}$} \\
\hline & & $\mathrm{H}_{2}$ & & $\mathrm{CO}_{2}$ & $\mathrm{CH}_{4}$ & $\mathrm{C}_{2} \mathrm{H}_{6}$ & $\mathrm{C}_{2} \mathrm{H}_{4}$ & $\mathrm{C}_{3} \mathrm{H}_{8}$ & $\mathrm{C}_{3} \mathrm{H}_{6}$ & $\mathrm{C}_{4} \mathrm{H}_{10}$ & $\mathrm{C}_{4} \mathrm{H}_{10}$ \\
\hline $407,408,409$ & 39 & $\frac{1.222(95.62)}{0.994}$ & $\frac{98.84}{87.56}$ & - & $\frac{0.045(3.84)}{0.040}$ & $\frac{0.0061(0.53)}{0.0055}$ & $\frac{-}{0.0161(4)}$ & $\frac{-}{0.0074(17)}$ & $\frac{-}{0.0363(2)}$ & $\frac{-}{0.0363(2)}$ & $\frac{-}{0.0162(4)}$ \\
\hline $410,410 \mathrm{~A}$ & 13 & $\frac{1.31(95.84)}{0.812}$ & $\frac{98.38}{48.49}$ & $10 . \overline{87}(1)$ & $\frac{0.045(3.81)}{0.022}$ & $\frac{0.005(0.35)}{0.0025}$ & $\frac{-}{0.0023(1)}$ & $\frac{-}{0.0019(3)}$ & - & - & - \\
\hline $411,412 \mathrm{~A}, 413$ & 28 & $\frac{0.498(93.96)}{0.422}$ & $\frac{99.47}{84.26}$ & - & $\frac{0.027(5.09)}{0.023}$ & $\frac{0.005(0.35)}{0.0014}$ & $\frac{-}{0.0011(3)}$ & $\frac{-}{0.0014(8)}$ & $\frac{-}{0.0004(2)}$ & $\frac{-}{0.0019(2)}$ & $\frac{-}{0.0012(1)}$ \\
\hline
\end{tabular}

Note: In the numerator, in brackets: chemical composition of gases without nitrogen, \%. In the denominator, in brackets: number of samples, the average content of a component was calculated from. 

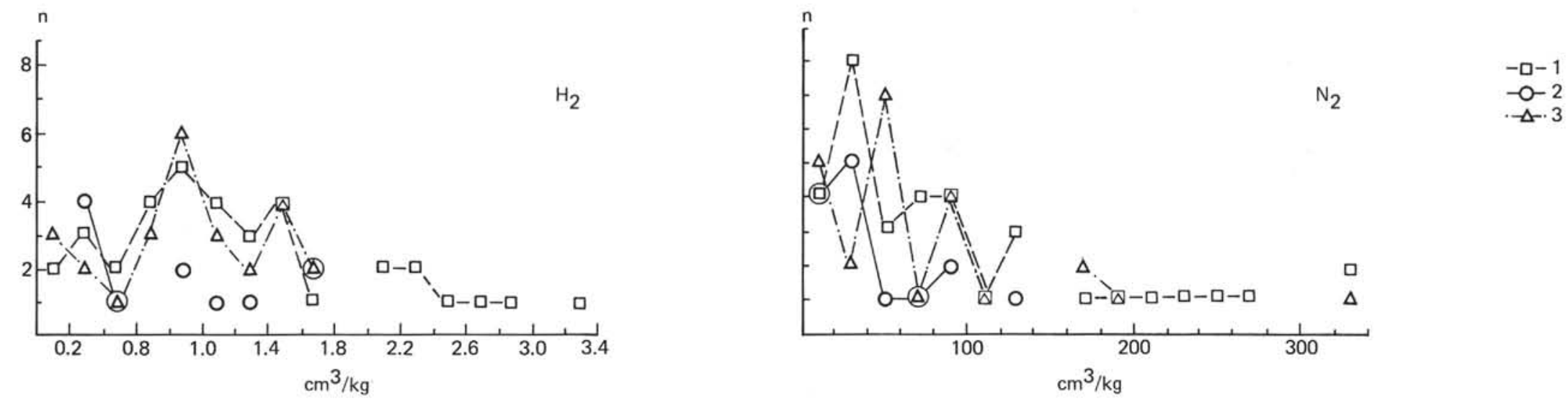

$-0-2$
$-\Delta .3$
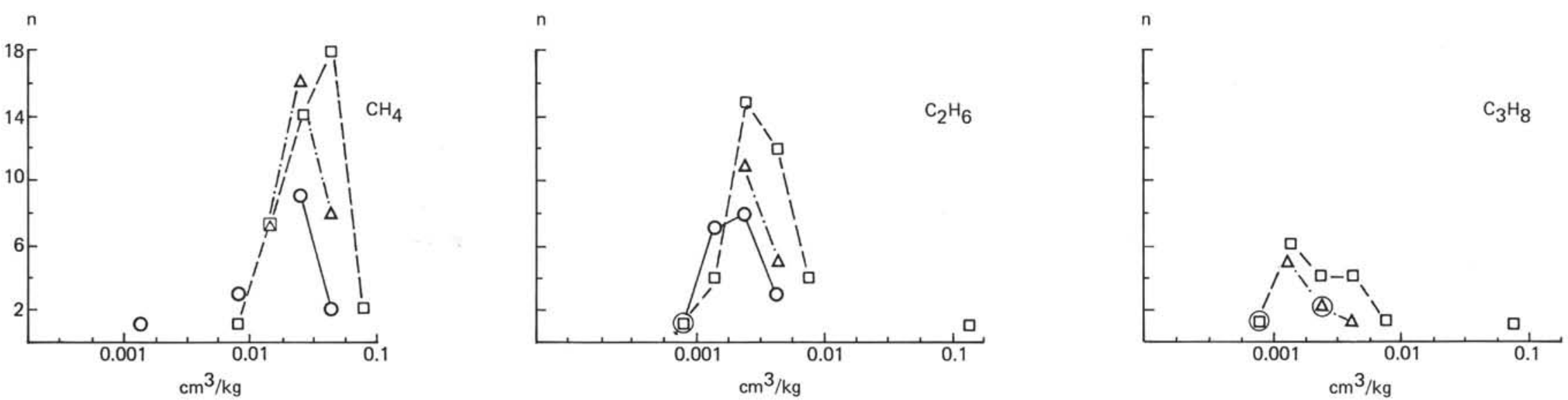

Figure 14. Histogram of gas distribution. Symbols as in Figure 3. 